\title{
The Aptamer Functionalized Nanocomposite Used for Prostate Cancer Diagnosis and Therapy
}

\author{
Xiaoqian Feng, ${ }^{1,2}$ Yinxing Zhu $\mathbb{D}^{3}{ }^{3}$ Fujin Wang, ${ }^{1}$ Ting Guo, ${ }^{4}$ Xiaofeng Dou, ${ }^{1}$ Mei Lin $\mathbb{D},{ }^{5}$ \\ and Weizhong Tian iD \\ ${ }^{1}$ Medical Imaging Department, Taizhou People's Hospital Affiliated to Nantong University, Taizhou, Jiangsu 225300, China \\ ${ }^{2}$ Department of Radiology, the First People's Hospital of Yancheng, Yancheng, Jiangsu, China \\ ${ }^{3}$ Faculty of Graduate Studies, Nanjing University of Chinese Medicine, Nanjing, 210023 Jiangsu, China \\ ${ }^{4}$ Institute of Clinical Medicine, Taizhou People's Hospital Affiliated to Nantong University, Taizhou, Jiangsu 225300, China \\ ${ }^{5}$ Clinical Laboratory, Taizhou People's Hospital Affiliated to Nantong University, Taizhou, Jiangsu 225300, China
}

Correspondence should be addressed to Mei Lin; 1_mei@163.com and Weizhong Tian; tz_twz@163.com

Received 22 March 2021; Revised 29 September 2021; Accepted 15 November 2021; Published 11 January 2022

Academic Editor: Vidyadhar Singh

Copyright (C) 2022 Xiaoqian Feng et al. This is an open access article distributed under the Creative Commons Attribution License, which permits unrestricted use, distribution, and reproduction in any medium, provided the original work is properly cited.

\begin{abstract}
Prostate cancer is one of the major malignancies that threaten men's health all over the world. Due to the lack of specific symptoms and signs in the early stage, as well as the limitations of existing detection methods, it is difficult to achieve early diagnosis for prostate cancer. As short single-stranded oligonucleotides (DNA or RNA) with specific 3D structure which can be produced using an in vitro selection process termed systematic evolution of ligands by exponential enrichment (SELEX), aptamers can specifically bind to the corresponding targets. They have become a class of novel targeting ligand for accurate diagnosis and effective treatment of cancer. Owing to distinctive physicochemical features, and some other special properties such as easy modifiability, good biocompatibility, being easily coupled with other ligands, nanomaterials are extensively used in biological medical field research. Enlighteningly, the combination of aptamers with nanomaterials, including metal nanoparticles, nanosilica, quantum dots, and carbon nanomaterials, can enhance the ability of nanomaterials to recognize tumor cells, which is beneficial to overcome the shortcomings such as low sensitivity in early detection and lack of specificity of traditional antineoplastic drugs, thus, clinically helpful to improve the early metaphase diagnosis rate, providing a technical guarantee for the "personalized treatment" strategy for prostate cancer. Herein, we mainly review the basic and applied research of aptamer functionalized nanocomposite in prostate cancer diagnosis and treatment, including biosensing, bioimaging, and cancer therapy, hoping to provide new ideas for prostate cancer diagnosis and treatment.
\end{abstract}

\section{Introduction}

Prostate cancer $(\mathrm{PCa})$ is a urinary malignant tumor with a very high incidence, and its mortality accounts for about $10 \%$ of all tumor-related death cases [1]. Typically, PCa is especially common among elder men in Europe and the United States. Because of the lack of early specific symptoms of PCa and the low sensitivity and specificity of existing detection methods, many patients are in the advanced stage at the time of initial treatment and are thus deprived of the optimal treatment time [2]. Therefore, early diagnosis and timely and effective treatment are of great significance to reduce the mortality and improve the life quality of PCa patients. As a new type of recognition molecules, nucleic acid aptamers have a great application potential in the research fields of tumor diagnosis and treatment [3] and have aroused wide attention from scholars at home and abroad. Meanwhile, with the continuous innovation of nanopreparation technology, it is possible for the emergence of high-performance nanomaterials with definite and controllable particle size and more complex functions [4]. The modified nanomaterials have excellent biocompatibility and strong targeting ability, which are extensively used in research in the biological medical field [5]. In this review, 
the research progress of aptamer-nanocomposites as a novel molecular tool to enhance the recognition and detection of prostate cancer cells is reviewed, and the exploration of use of aptamer functionalized nanomaterial in early diagnosis and treatment for prostate cancer is overviewed.

\section{Aptamers}

Aptamer, a kind of short single-stranded oligonucleotide sequence, can specifically bind to all kinds of target molecules via complementary spatial configuration. Aptamer was first screened by Ellington, Szostak, Tuerk, and Gold as early as 30 years ago $[6,7]$, and it can be produced by SELEX today. The conventional SELEX process mainly involves the following three steps: selection, partitioning, and amplification (Figure 1).

Due to its conformational change or special threedimensional structures, such as hairpin, internal loops, pseudoknot, convex ring bulge, and G-tetramer [8], aptamers can bind to the corresponding target molecules with high selectivity and affinity under the action of noncovalent bonds such as static electricity, van der Waals force, and hydrogen bond [9]. With a broad range of targets, aptamer targets can be small organic or inorganic molecules, peptides, proteins, tissues, cells, viruses, or even parasites. Since the binding process of aptamers to targets is similar to the binding reaction of antigen with antibody, it is also called "chemical antibody." Compared with traditional antibodies, aptamers are associated with the advantages of low molecular weight, lack of immunogenicity, high thermal stability, easy synthesis, and modification. As a result, they have become the ideal candidates for molecular probe in the field of cancer and have been widely used in the targeted diagnosis and treatment of tumors, particularly in the fields of biosensor, in vitro and in vivo imaging, or targeted delivery therapeutics $[10,11]$.

\section{Specific Aptamers against Prostate Cancer and the Related Targets}

Since the development of SELEX, aptamers have been extensively utilized in various studies, including the exploration of new diagnostic tools and therapeutic methods for tumors. Different from conventional SELEX (protein-based SELEX), cell-SELEX refers to the use of whole cells living in vitro as a screening target to obtain aptamers specifically binding to target cells by making use of the differences in proteins or structures on the surface of different cells [12]. There is no need to have the prior knowledge of the molecular targets on the cell surface before selection. The aptamers screened by this method can recognize cells under different physiological and pathological conditions according to the changes in cell membrane surface molecules, which provide a basis for disease diagnosis and treatment at the molecular level [13].

The vital segment in the aptamer discovery process is whether to select for DNA or RNA aptamers. While both types of aptamers have similar characteristics and been proven to be effective in a wide range of applications, there are some key differences in terms of chemical stability and target accessibility [14]. Early aptamer studies focused primarily on RNA, and most of the classical aptamers for PCa belong to RNAs [15]. Native DNA aptamers are more stable than RNA aptamers due to the absence of a reactive hydroxyl group, which is deprotonated in alkaline solutions triggering their hydrolysis, so DNA aptamers are relatively less reactive, making their easier storage and processing than those of RNA aptamers [16].

As shown in Table 1, so far, a large number of studies have reported the synthesis of aptamers targeting PCa.

\subsection{RNA Aptamer Targeting the Prostate-Specific Membrane} Antigen (PSMA). PSMA, a type II integral membrane glycoprotein, has been considered as an attractive target for the diagnosis and treatment of PCa. PSMA is lowly expressed in some tissues, but its expression level is only one percent to one specificity thousandth in PCa tissue, indicating that PSMA is highly specific to prostate tissue or organ [29]. Moreover, the expression of PSMA is independent of the androgen level in the body, which remains high during all stages of the disease. This is of great significance for patients who develop the hormone-refractory PCa after endocrine therapy [30]. The first generation of classic aptamers for PCa is RNA aptamers A9 and A10 [17]. This series of aptamers take the PSMA, which specifically binds to the PSMApositive PCa cells, as the target protein and are then ingested into the cells. Subsequently, after a series of modification and optimization, aptamers have been also used in some truncated forms such as A10-3, A10-3.2 [19], and A9g [18], respectively. Considering the more diverse threedimensional structures of RNA aptamers, the affinity of A9 and A10 aptamers is improving after their truncations. Simultaneously, the relevance of PSMA as a PCa target is well known. Given the above, A9 and A10 aptamers stand out due to their performance in PCa theranostics.

3.2. DNA Aptamer Targeting Prostate-Specific Antigen (PSA) and Androgen Receptor (AR). The serum PSA has been the preferred biomarker for the detection and monitoring of PCa over the past decades [31]. However, high levels of PSA are not necessarily related to the cancerous state of $\mathrm{PCa}$, which may fluctuate due to the inflammation, infection, or benign prostatic hyperplasia $(\mathrm{BPH})$, thus, resulting in misdiagnosis and overtreatment of indolent PCa [32]. Notwithstanding its own limitations, PSA remains the most extensively reported biomarker, possibly due to its substantial use in the current clinical practice of PCa. Consequently, it is crucial to develop more ultrasensitive and highly accurate methods to detect the PSA concentration [33].

A DNA aptamer against PSA using genetic algorithms was recognized by Savory and coworkers in 2010. This is the first report of a DNA aptamer against PSA; meanwhile, several methods have also been proposed using aptamerbased PSA biosensing, including electrochemical techniques or optical [26]. Wong et al. [34] optimised the ratio of antiPSA DNA aptamer with 6-mercapto-1-hexanol (MCH) and used gold electrodes to fabricate the aptasensor, structuring a sensitive label-free, cost-effective system with simultaneous 


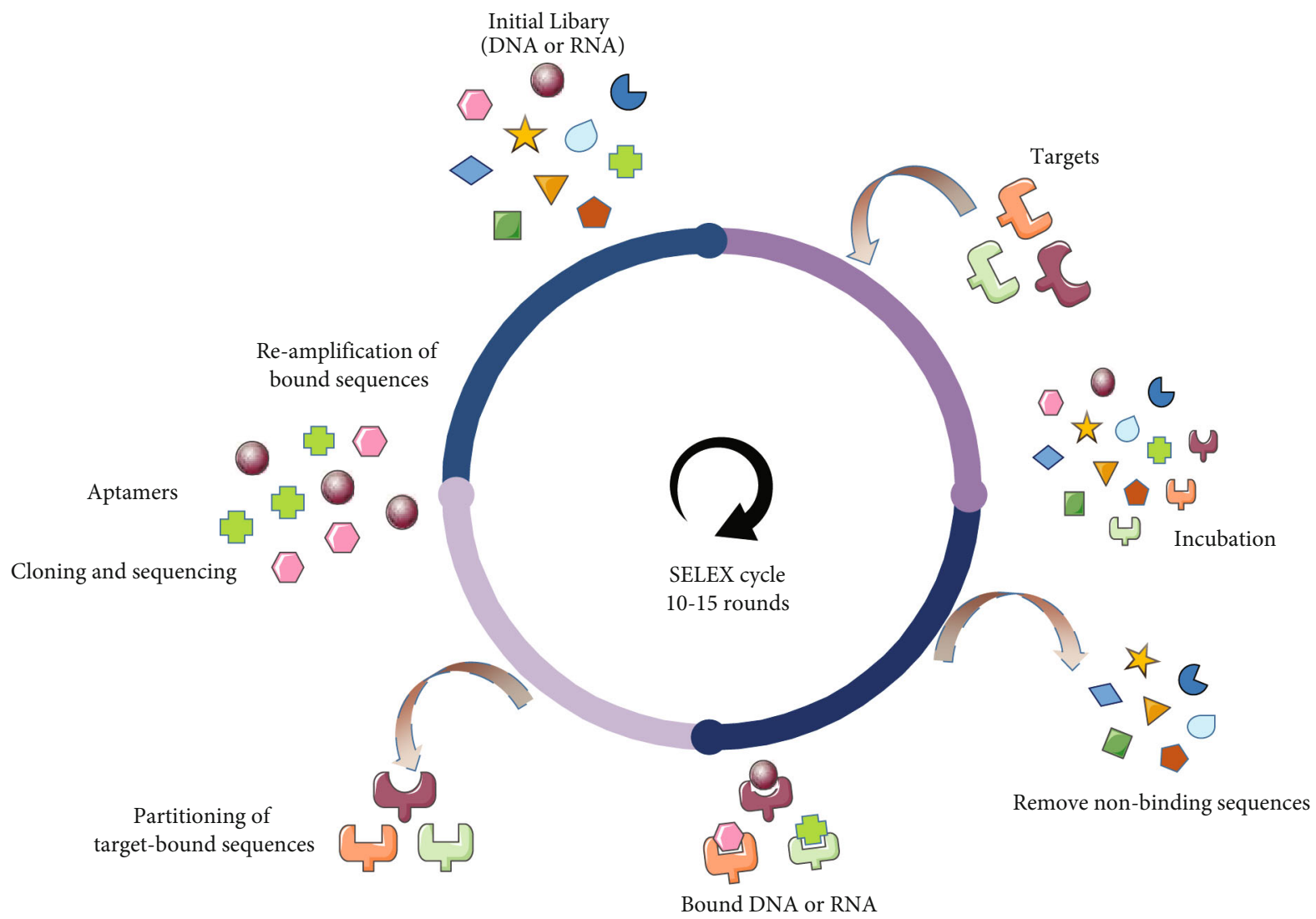

FIgURE 1: Schematic illustration of aptamer selection by systematic evolution of ligands by exponential enrichment (SELEX). The starting single-stranded DNA or RNA library $\left(10^{13} \sim 1015\right.$ random oligonucleotides $)$ is composed of sequences $20 \sim 100$ nucleotides in length. Each unique sequence contains random bases (30-50 nt) flanked by two conserved primer binding sites, which are used for PCR amplification by annealing primers. After incubation with the target of interest, the bound oligonucleotides are partitioned from unbound sequences. The target-bound sequences are amplified by PCR, and the resulting new subpool is utilized for the next round of selection.

TABLE 1: Specific aptamers against prostate cancer.

\begin{tabular}{lccc}
\hline Aptamer names & Targets & Types & Ref. \\
\hline A9, A10, A10-3 & PSMA & RNA & Lupold et al., 2002 [17] \\
A9g, A10L & PSMA & RNA & Rockey et al., 2011 [18] \\
A10-3.2 & PSMA & RNA & Dassie et al., 2009 [19] \\
A10-3-J1 & PSMA & RNA & Leach et al., 2016 [20] \\
Wy-5a & PC-3 & DNA & Wang et al., 2014 [21] \\
DML-7 & PC-3 and DU-145 & DNA & Duan et al., 2016 [22] \\
AMH & DBD (DNA-binding) of AR & DNA & Kouhpayeh et al., 2016 [23] \\
CSC1, CSC13 & DU-145 cells DU-145 stem cells & Wang et al., 2013 [24] \\
E3 & LNCaP, 22Rv1, PC-3, and DU-145 cells & DNA & Gray et al., 2018 [25] \\
PSap4\#5 & PSA & DNA & Savory et al., 2010 [26] \\
S3.1/S2.2 & MUC-1 & DNA & Karpik et al., 2017 [27] \\
AS1411 & Nucleolin & DNA & Bates et al., 2009 [28] \\
\hline
\end{tabular}

multichannel measurement of open circuit potential (OCP) variations for PSA detection. Chen et al. [35] recently have designed a novel PSA detective sensors based on the aptamer recognition and enzyme-assisted target recycling (EATR) amplification. By introducing two functional DNA probes (PSA-aptamer and a poly (T) Taqman probe labeled with both fluorophore and quencher), this highly sensitive and cost-effective aptasensor gave an excellent signal and proved its considerable potential for practical assay.

ARs have a crucial role in the function and homeostasis of PCa cells; it can be activated as a transcription factor via binding to androgen hormones [36]. The receptor has two 
essential functional domains: DNA-binding (DBD) and ligand-binding. Kouhpayeh et al. [23] designed a singlestranded DNA as an aptamer that could simulate the hormone response element (HRE). It seems that the aptamer mimicking HRE is capable of competing with native HRE on genomic DNA and reduced the effect of the androgen hormone complex to induce transcription of related genes. The synthetic aptamer could be a powerful tool for gene therapy of advanced PCa that may no longer respond to traditional hormone deprivation therapy.

3.3. DNA Aptamer Targeting PCa Cells. In addition to target protein, some aptamers can selectively recognize and bind to target cells, such as LNCaP, 22Rv1, PC-3, and DU-145, the well-established human PCa cell lines [25]. Typically, LNCaP has a high sensitivity to androgen, while $22 \mathrm{Rv} 1$ has a low sensitivity to androgen, and the others are the androgen non-reliant cell lines. Wang et al. [21] screened a DNA aptamer probe $\mathrm{Wy}-5 \mathrm{a}$, for prostate cancer against PCa cell line PC-3. Wy-5a showed high specificity to the target cells with dissociation constants in the nanomolar range, but did not recognize other tested PCa cell lines or other tumor cell lines. The staining of clinical tissue sections with fluorescent dye-labeled $\mathrm{Wy}-5 \mathrm{a}$ shows that sections from high-risk group with metastasis exhibited stronger fluorescence and no notable fluorescence in sections from $\mathrm{BPH}$, which suggests that aptamer Wy-5a could be used to distinguish between $\mathrm{BPH}$ and high-risk $\mathrm{PCa}$ specimens. Duan et al. [22] selected a DNA aptamer termed DML-7, which can selectively recognize and bind to prostate cancer cells (PC-3 and DU-145) that do not express AR, but not to AR-positive prostate cancer cells (LNCaP and 22RV1). In addition, Drabik and her team focused on the unambiguous identification of the molecular targets and aptamers A26/A33 were screened out, which have excellent selectivity for the PC-3luc prostate cancer cell line [37].

3.4. Other Targets Related to PCa. There are other studies reporting membrane proteins such as MUC-1, an important biomarker for cancer detection related to the metastasis mechanisms and overexpressed in most adenocarcinomas [38]. Studies have shown that MUC-1 is found in $60 \%$ of $\mathrm{PCa}$ cells, and its expression significantly increases in advanced $\mathrm{PCa}$ (both during the metastasis and progression of castration-resistant prostate cancer (CRPC)) [27], so it represents an attractive target. Nucleolin works as a nuclear biomarker, which is involved in cell proliferation and overexpressed in cancer cells, both intracellularly and on the cell surface, such as PCa, lung cancer, and breast cancer (BC) [28]. AS1411 aptamer is a guanine-rich oligonucleotide that specifically binds to nucleolin with high affinity, which has been verified to exert the antiproliferative and cytotoxic effects on cancer cells [39], owing to a novel nonapoptotic cell death-methuosis. As both the therapeutic aptamer and nucleolin target, AS1411 has a great potential in the detection and treatment of $\mathrm{PCa}$, as shown in Table 2.

\section{Nanomaterials Linked to Aptamers and Main Biomedical Applications}

Aptamers display a high variety of refinements and can be readily designed or chemically evolve to enhance their performance in theranostic applications [42].

It is well known that nanoparticles (NPs) are biocompatible and usually around $10-30 \mathrm{~nm}$ in diameter; typically, their smaller size and high surface-area-to-volume ratio can achieve high accumulation in tumor tissue via the enhanced permeability and retention (EPR) effect [43]. The modifiable feature of NPs also renders them a superior targeting ability once a suitable ligand is conjugated. In addition, nanomaterials exhibit fantastic properties in magnetism, electricity, optics, and catalysis [44]. As a result, models constructed by aptamers linking to NPs have a great application prospect, although their clinical application is rarely reported, great progresses have been achieved in experimental studies at the cellular and animal levels.

The frequently used nanomaterials include liposomes, polymerNPs, dendrimer, quantum dots (QDs), carbon nanomaterials, nanosilica, and magnetic NPs. It is important to analyze the characteristics of nanomaterials to guide their practical application. (Figure 2, Table 3).

\section{Application of Aptamer-Nanocomposites in the Diagnosis of Prostate Cancer}

PCa brings huge health and economic burdens to the society. Due to the increased case number and the new therapeutic option of $\mathrm{PCa}$, the traditional clinical diagnostic mode has been criticized for the overdiagnosis for low-risk $\mathrm{PCa}$ and the underdiagnosis for clinically significant PCa [59]. Today, precision medicine has been closer to the reality; thus, it is extremely important to explore new methods to effectively diagnose $\mathrm{PCa}$ and improve the early diagnosis rate.

5.1. Detection of Prostate Cancer Biomarkers. As we all know, biomarker detection is of great reference significance to the screening, risk stratification, and relapse monitoring of PCa [60]. As incredible progresses have been made in nanotechnology, biosensors based on the principles of fluorescence [61], colorimetry [62], electrochemical [63], and signal amplification [64] have been widely applied in the biomedical field due to their advantages of good selectivity, high sensitivity, rapid analysis, and low cost [65]. Aptamers, which can selectively bind to tumor-related molecules on cell surface, display distinct superiorities over traditional antibodies by virtue of their unique biological properties. It is expected that related aptamers can be used in combination with nanobiosensor to effectively detect the biomarkers at low concentrations for $\mathrm{PCa}$, which thus greatly improves the early diagnosis rate of $\mathrm{PCa}$ and has an important practical value [66].

By using the mesoporous silica thin film-coated gold electrodes combined with anti-PSA specific DNA aptamers, Argoubi et al. [54] reported the development of a novel label-free electrochemical aptasensing platform to detect 
TABLE 2: Part of prostate cancer cell-specific aptamers sequences.

\begin{tabular}{|c|c|}
\hline Aptamer names & Aptamer sequence $\left(5^{\prime}-3^{\prime}\right)$ \\
\hline A9 & $\begin{array}{l}\text { GGGAGGACGAUGCGGACCGAAAAAGACCUGACUUCUAUACUAAGUCUACGUUCCCAGACGACUCGC } \\
\text { CCGA [17] }\end{array}$ \\
\hline A10 & GGGAGGACGAUGCGGAUCAGCCAUGUUUACGUCACUCCUUGUCAAUCCUCAUCGGC [40] \\
\hline A10-3.2 & GGGAGGACGAUGCGGAUCAGCCAUGUUUACGUCACUCCU [19] \\
\hline Wy-5a & TGCCACTACAGCTGGTTCGGTTTGGTGACTTCGTTCTTCGTTGTGGTGCTTAGTGGC [21] \\
\hline DML-7 & $\begin{array}{l}\text { ACGCTCGGATGCCACTACAGGTTGGGGTCGGGCATGCGTCCGGAGAAGGGCAAACGAGAGGTCACC } \\
\text { AGCACGTCCATGAG [22] }\end{array}$ \\
\hline $\mathrm{CSC} 1$ & $\begin{array}{l}\text { ACCTTGGCTGTCGTGTTGTAGGTGGTTTGCTGCGGTGGGCTCAAGAAGAAAGCGCAAAGGTCAGTG } \\
\text { GTCAGAGCGT [24] }\end{array}$ \\
\hline $\mathrm{CSC} 13$ & $\begin{array}{l}\text { ACCTTGGCTGTCGTGTTGTGGGGTGTCGTATCTTTCGTGTCTTATTATTTTCTAGGTGGA GGTCAGTGG } \\
\text { TCAGAGCGT [24] }\end{array}$ \\
\hline AMH & GCCGTATGGTACACGGTGTTCTAAACTATAAGAACACCGTGTACCATACGGC [23] \\
\hline $\begin{array}{l}\text { S2.2 anti-MUC1 } \\
\text { aptamer }\end{array}$ & CAGTTGATCCTTTGGATACCCTG [41] \\
\hline AS1411 & GGTGGTGGTGGTTGTGGTGGTGGTGG [40] \\
\hline
\end{tabular}

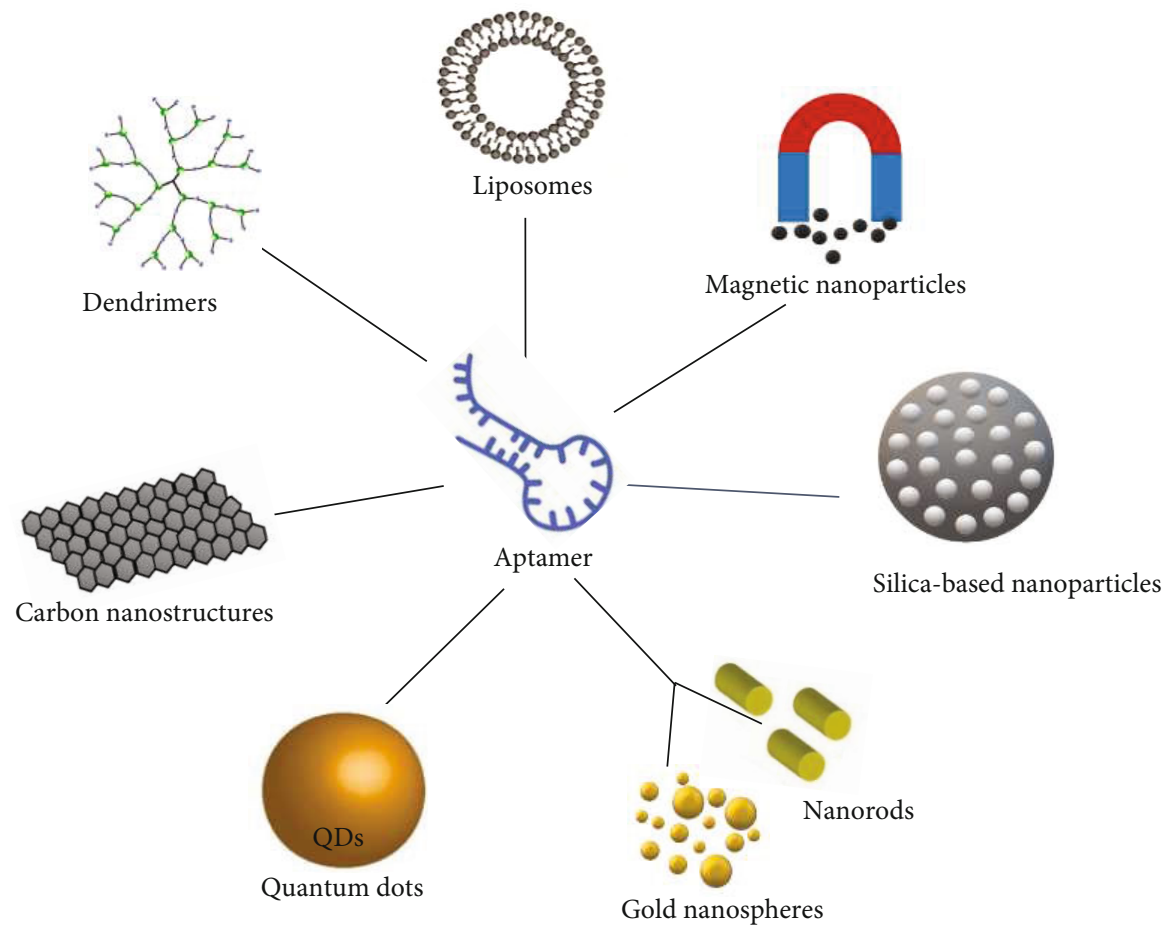

FIGURE 2: Various principal nanomaterials linked to aptamers. This figure depicts the most common types of nanomaterials linked to aptamers, including carbon nanostructures, polymeric nanocarriers, lipid-based nanocarriers, magnetic nanoparticles, silica-based nanoparticles, semiconductor nanoparticles, and plasmonic nanocarriers.

PSA. The aptasensor showed high sensitivity, specificity, repeatability, and storage stability for PSA and was validated in the spiked artificial urine and in blood serum samples from a PCa-free male patient. It is an important key to use biocompatible organic macromolecules in combination to enhance the system sensitivity and stability in the immunosensor development field [67]. Kavosi et al. [68] developed an ultrasensitive fluorescence assay to detect PSA by the combined use of CdTe QDs and aptamer-PAMAM-
AuNPs. In their study, PAMAM dendrimers with multiple functional groups were used to make a large surface area for the effective immobilization of PSA aptamer. This proposed immunosensor attained an outstanding analytical performance towards PSA, which may be applied in detecting the trace level of PSA and supplying valuable information for the early clinical diagnostics.

Some studies combined PSA with several other biomarkers such as vascular endothelial growth factor (VEGF) 
TABLE 3: Summary of aptamer-conjugated nanomaterials for cancer diagnosis/therapy.

\begin{tabular}{|c|c|c|c|}
\hline Nanoparticles & Category & Aptamer & Application \\
\hline $\begin{array}{l}\text { Carbon nanostructures (nanotube, } \\
\text { graphene) }\end{array}$ & Organic & $\begin{array}{c}\text { The anti-PSMA specific RNA } \\
\text { aptamer, AS1411 }\end{array}$ & Bioimaging [45], biosensing, drug delivery [46] \\
\hline $\begin{array}{l}\text { Polymeric nanocarriers (dendrimers, } \\
\text { polymeric micelles) }\end{array}$ & Organic & $\begin{array}{c}\mathrm{A} 10 \\
\mathrm{~A} 10-3.2\end{array}$ & $\begin{array}{l}\text { Drug delivery [47], molecular imaging [48], } \\
\text { gene therapy [49] }\end{array}$ \\
\hline Lipid-based nanocarriers (liposomes) & Organic & $\begin{array}{c}\mathrm{A} 10 \\
\mathrm{~A} 10-3.2\end{array}$ & $\begin{array}{l}\text { Drug delivery, molecular imaging [50], gene } \\
\text { therapy [51], }\end{array}$ \\
\hline Magnetic nanoparticles & Inorganic & $\begin{array}{c}\text { A10 } \\
\text { A10-3-J1 } \\
\text { MA3 }\end{array}$ & MRI [52], MIH [53], drug delivery \\
\hline Silica-based nanoparticles & Inorganic & $\begin{array}{c}\text { The anti-PSA specific DNA } \\
\text { aptamer }\end{array}$ & $\begin{array}{c}\text { Multimodal bioimaging, drug release, PDT, } \\
\text { biosensing [54] }\end{array}$ \\
\hline Semiconductor nanoparticles (QDs) & Inorganic & $\begin{array}{l}\text { The MUC1 aptamer-(CGA) })_{7} / \mathrm{S} 3.1 / \\
\text { S2.2 }\end{array}$ & Bioimaging, biosensing, and drug delivery [55] \\
\hline $\begin{array}{l}\text { Plasmonic nanocarriers (gold } \\
\text { nanospheres, nanorods) }\end{array}$ & Inorganic & $\begin{array}{c}\text { A9 } \\
\text { Sgc8c } \\
\text { CSC1/CSC13 }\end{array}$ & $\begin{array}{l}\text { Bioimaging [56], PTT }[57,58] \text {, plasmonic } \\
\text { sensors, gene therapy }\end{array}$ \\
\hline
\end{tabular}

*MIH: magnetic induced hyperthermia; PDT: photodynamic therapy; PTT: photothermal therapy.

[69], carcinoembryonic antigen (CEA) [70], and epithelial cell adhesion molecule (EpCAM) [71] to improve the precision of PCa diagnosis. The monitoring of VEGF is widely applied to detect various cancer types, including urinary tumor. In order to overcome the shortcomings of PSA, VEGF has been studied as an alternative or additional cancer marker [72]. Chong et al. [73] devised a dual aptasensor based on the principle of guanine chemiluminescence reaction for the early diagnosis of prostate cancer. They further confirmed that the aptasensor can be applied as a new screening tool capable of simultaneously quantifying PSA and VEGF in a sample with good accuracy, precision, and reproducibility. CEA is also one of the most important tumor markers, and it shows a measurable clinical value for the early stage diagnosis of neoplasms. Hence, establishing an ultrasensitive medical device for the simultaneous detection of CEA and PSA is of great significance for the accurate prediction of prostate cancer [74]. Using fluorometric assay based on the synergistic effect of fluorescence resonance energy transfer (FRET) and internal filter effect (IFE), Sun et al. [75] fabricated a dual detection fluorescence biosensor composed of mesoporous silica nanoparticles (MSNs) loaded with CdTe QDs for the rapid detection of both CEA and PSA. The unique porous structures and high specific surface area enable MSNs to carry numerous QDs, which further amplify the fluorescence signal. To warrant the selectivity of assay, two aptamers were covalently connected to fluorescent MSNs as the recognition unit. It is illustrated that the sensor successfully achieved sensitive and accurate simultaneous detection of CEA and PSA, which may be applied for an effective tool to aid designing PCa personalized therapeutic protocols. From the above, the incorporation of the PSA into multiplexed designs with other biomarkers has proven to be highly necessary and valuable for clinical diagnosis and prognosis of prostate cancer.

5.2. Molecular Imaging of Prostate Cancer. With the rapid development of nanomedicine [76], molecular biology, poly- mer science, and other related disciplines over the last few years, a variety of new technologies have been applied in the field of medical imaging. The traditional imaging patterns in tumor diagnosis mainly focusing on the anatomical structure are quietly changing $[77,78]$. As a newly developed diagnostics, molecular imaging has dramatically evolved technologically in the field of medical imaging [79]. It aims to specifically target different biological properties of the tissue and provide information on key pathophysiological processes involved in diseases at molecular and cellular levels, so as to address the diagnostic gaps in precision medicine [80]. The application of molecular imaging in clinic contributes to the early diagnosis before any tiny structural changes are detected by traditional ways, and this will bring a new breakthrough for the diagnosis and treatment of PCa [81].

5.2.1. Magnetic Resonance Imaging (MRI) with Aptamers. As a powerful noninvasive diagnostic modality, MRI exhibits several advantages, such as high soft-tissue resolution and avoidance of ionizing radiation; in addition, it can provide multiparameter, multisequence three-dimensional images and has a high potential in molecular imaging [82]. Gadolinium- (III-) based contrast agents (GBCAs) are routinely used to enhance the contrast between lesions and surrounding normal tissues on MRI images, which have played an indispensable role in facilitating the diagnosis of PCa. Even so, the clinical application of GBCAs is limited to a great extent due to their potential dose-dependent toxicity and nonspecific organ accumulation [83]. In this regard, it is imperative to meet the needs of clinical practice by developing new type tumor-specific targeted contrast agents. MRI scanning with high spatiotemporal resolution and abundant oncogenic biomarkers in tumor microenvironment [84] have been enabled to provide precision cancer imaging. To overcome the defects of traditional MR contrast agents, researchers have developed several novel nanoscale contrast agents, many of which have acted as the carriers of targeted contrast agents and then become a part of the functional or 
actively targeted nanoprobes $[85,86]$. Once entering the body, these multifunctional molecular probes conjugated with related tumor-targeted biomolecules are expected to be directly located at the target sites through specific binding, such as antigen-antibody reactions [87] and ligandreceptor reactions [88], thereby inducing changes in the local MR signal (Figure 3).

In recent years, a series of magnetic nanoprobes modified with aptamers have been applied in the MRI molecular imaging for cancer detection. All of them significantly improve the tumor-targeting ability and biocompatibility, reduce the cytotoxicity, and further enhance the imaging quality of MRI. Yu et al. [89] conjugated PSMA-aptamers with the thermally cross-linked superparamagnetic iron oxide nanoparticles (TCL-SPIONs) via a hybridization method. Subsequently, the authors used the nanocomposites as a specific contrast agent for the research on MRI molecular imaging of PCa. When analyzed by $T_{2}$-weighted MRI, the APT-TCL-SPION nanocomposites exhibited preferential binding towards PCa cells overexpressing PSMA both in vitro and in vivo. These results proved that it had the potential for use as a novel targeted MR contrast agent for PCa molecular imaging.

5.2.2. Ultrasound Imaging. Ultrasound imaging, one of the most commonly-used imaging techniques in clinics, has the advantages of low cost, portability, wide availability, outstanding safety profile, and once associated with targeted ultrasound contrast agents (UCAs), can identify the molecular expression of cancer-related biomarkers, which widens the range of clinical application and makes ultrasonic molecular imaging possible [90]. Nanobubbles, as a kind of new UCAs, which can break through the limitation of conventional UCAs, have shown a promise to penetrate tumor vasculature for extravascular molecular imaging due to their unique nanoscale size and high biocompatibility [91]. Recently, several kind of targeted nanobubbles with various shells such as phospholipids or polymers have been successfully developed. They used EPR effects of tumor vasculature to enter the tissue space and further combined with tumor cells by specific ligands taken with themselves, thus, achieving the effect of noninvasive and continuous monitoring tumors at the cellular or molecular levels (Figure 4) [92].

Fan et al. [50] linked fluorinated A10-3.2 aptamers with lipid molecules from the outer shell of nanobubbles via amide reaction, then successfully fabricated the lipidtargeted nanobubble carrying the anti-PSMA A10-3.2 aptamer and investigated its effect in the ultrasound imaging of prostate cancer. The results of in vitro binding experiments and flow cytometry showed that the targeted nanobubbles could bind with PSMA-positive C4-2 cells, while not with PSMA-negative PC-3 cells. In vivo experimental results indicated that targeted nanobubbles could specifically recognize the C4-2 prostate cancer xenograft with PSMA expression in the contrast-enhanced ultrasound mode. The experimental design not only provided ultrasound molecular probes with strong penetration and specificity for prostate cancer but also afforded references for relevant studies on targeted ultrasound nanobubbles carrying aptamers. Gu et al. [45] designed a new nanoultrasound contrast agent by modifying multiwalled carbon nanotubes (MWCNTs) with poly (ethylene glycol) (PEG) and anti-PSMA aptamer. The result revealed that the modified MWCNTs were able to target PCa cells more effectively as compared with the conventional contrast agent; in addition, it offered better visuality and veracity.

5.2.3. Nuclear Imaging and Multimodal Imaging with Aptamers. After initial curative treatment, such as radical prostatectomy or radiotherapy, up to $50 \%$ of PCa patients develop tumor recurrence, especially in patients with highrisk PCa [93]. However, several studies have shown that conventional morphological imaging are of extremely limited used in the detection of recurrent or/and metastatic disease. As another important mode of molecular imaging, positron emission tomography (PET) and single-photon emission computed tomography (SPECT) based on diagnostic radionuclides combine direct visualization of tumor dependent metabolism with morphological information [94], which provides a more reliable imaging information for the staging, metastasis, and recurrence of PCa. In addition, due to the heterogeneity of tumor cell clones and the unique microenvironment, a single targeted molecule and imaging technology is not enough to fully reflect the biological characteristics of prostate cancer [95]. Multimodal imaging can fuse the effective information from different imaging techniques, achieve complementary advantages to the greatest extent, and provide more accurate imaging information for tumor early diagnosis and treatment [96]. A combination of nanotechnology with optical, radionuclide, and MR imaging has a great potential to improve cancer diagnosis and therapy. Hwang et al. [97] constructed a nanoparticle-based multimodal tumor-specific imaging probe that can be simultaneously used for fluorescence imaging, MRI, and radionuclide imaging. In this system, aptamer AS1411 was used to enhance targeting and selectivity for imaging procedure. The multimodal targeting imaging system showed great specificity and stability both in vitro and in vivo imaging experiments. With a broad application prospect, the system may be used as a versatile imaging tool for specific cancer diagnosis. Kang et al. [98] designed a cancer-targeting multimodal imaging nanoplatform. The platform consisted of cobalt ferrite magnetic nanoparticles surrounded by fluorescent rhodamine within a silica shell and further conjugated with aptamer targeting underglycosylated MUC-1(uMUC-1). Subsequently, the nanocomposites were labeled by $68 \mathrm{Ga}$ with the help of a $p$-SCN-bn-NOTA chelating agent and finally successfully obtained the multifunctional imaging probe, which showed specific and dose-dependent fluorescent, radioisotope, MR signals targeting cells expressing uMUC-1 and might be a promising strategy for prostate cancer diagnosis shown in Figure 5.

\section{Application of Aptamer-Nanocomposites in the Therapy of Prostate Cancer}

Early and localized prostate cancer may be cured with surgery or radiation therapy, but the disease recurs in 

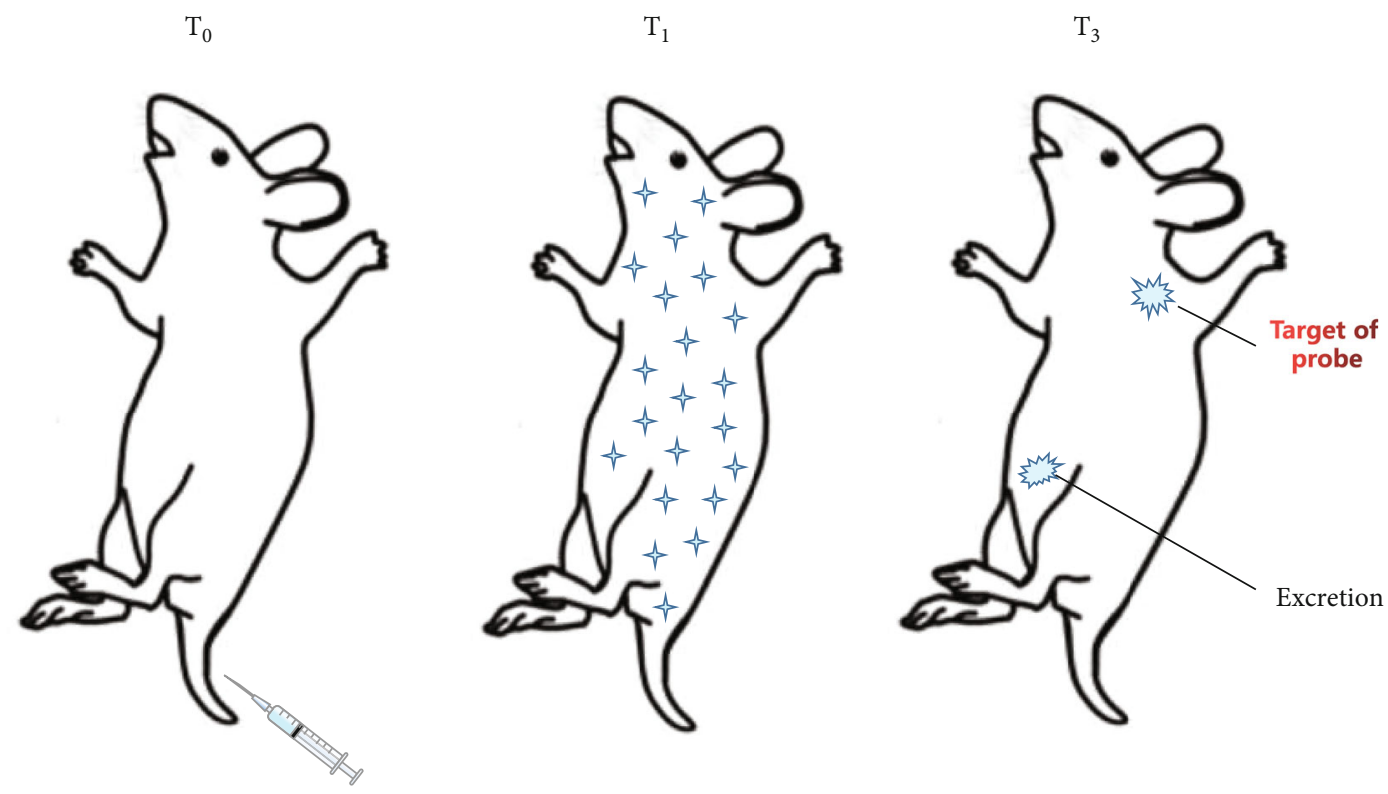

FIGURE 3: Principle of aptamer-based molecular imaging. For MRI imaging techniques, aptamers are linked to contrast agents based on nanoparticles to build imaging probes. At $T_{0}$, the contrast agent (in blue) is injected inside the subject. Then, it distributes throughout the body $\left(T_{1}\right)$ and is finally cleared from the tissues, except in the area of interest where the probe interacts with the target $\left(T_{2}\right)$.

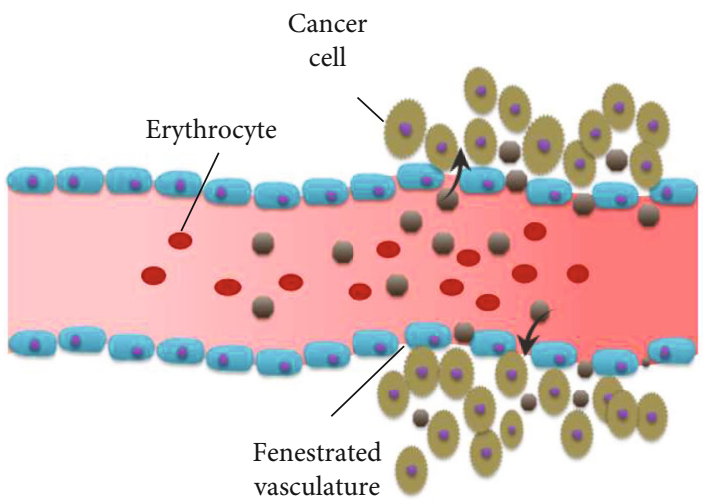

(a)

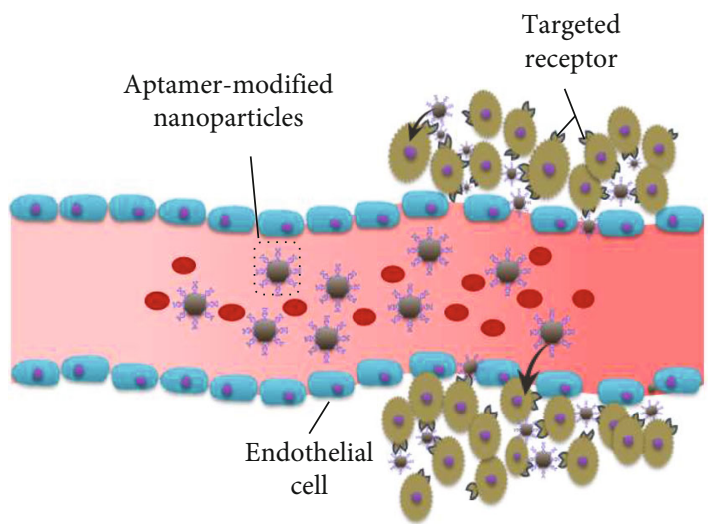

(b)

FIGURE 4: Schematic illustration of tumor-targeting nanoparticles. (a) Passive targeting. (EPR effect of nanoparticles). Extravasation of nanoparticles through fenestrated vasculature leads to passive targeting of tumor tissue; (b) active targeting. Ligands (aptamers, antibodies, small molecules, etc.) linked with nanoparticles that bind to receptors overexpressed by tumor cells.

approximately $30 \%$ of patients. Androgen-deprivation therapy is the standard treatment for advanced or recurrent prostate cancer; however, after a median remission period of 18-24 months, most patients who receive such treatment eventually develop into metastatic castration-resistant prostate cancer (mCRPC) $[99,100]$. Due to the lack of effective treatment, mCRPC is a disease with a poor prognosis which has become the most direct cause of death from terminal prostate cancer. In the last few years, despite the approval of several agents for mCRPC treatment, including cytotoxic drugs (cabazitaxel), second-generation antiandrogen compounds (abiraterone acetate) [101], and particles emitting radionuclides (radium-223) [102], has changed the natural history of this disease, prolonging survival and maintaining patients' life quality to some extent, mCRPC still remains a disease with a lethal outcome that requires new treatments to improve patients' outcome.

The rapid development of molecular imaging has brought new opportunities for clinical oncology, and cancer pathogenesis and therapy have thus been improved. Aptamer-nanocomposites are used in various fields of tumor therapy based on their physical and chemical properties, as well as the advantages in synthesis and production. They have shed novel light on the treatment for prostate cancer.

6.1. Aptamer Functionalized Nanocomposite in Chemotherapy of Prostate Cancer. Chemotherapy is one of the most commonly used therapeutic approaches for mCRPC; however, the clinical application of traditional chemotherapeutics has been largely limited by their low 


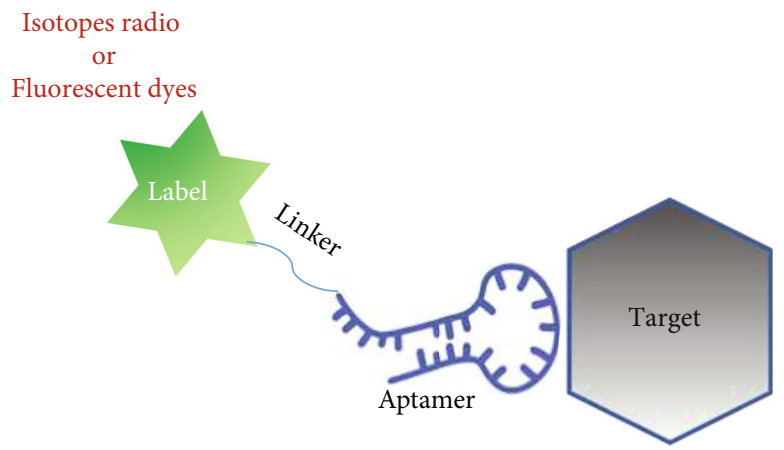

(a)

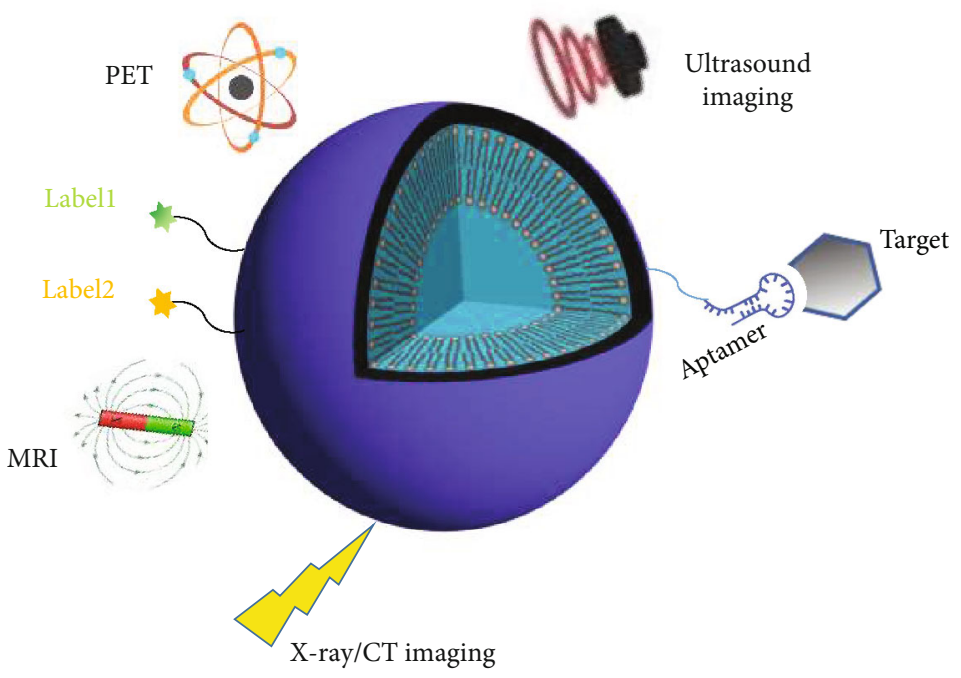

(b)

FIgURE 5: Schematic illustration of aptamer-based molecular imaging probes. The aptamer is conjugated by a linker to a label that allows detection by an imaging instrument. These probes generally used for nuclear imaging or fluorescence imaging. For MRI and other imaging techniques, aptamers are linked to contrast agents based on nanoparticles, which can be simultaneously labeled with several labels to build multimodal imaging probes.

selectivity to cancer cells and severe adverse reactions to the body [103]. The use of aptamer functionalized nanocomposites to deliver chemotherapeutics can effectively attenuate the damage to non-target cells and improve the bioavailability [104]. Similar to the antibody-drug conjugates that have been successfully used in targeted therapy against tumor, the aptamer-drug conjugates can recognize the targeting ligand of pathogenic sites or biomarkers, thereby specifically stimulating or inhibiting the corresponding target, and it is an ideal delivery system for target drugs [105]. In recent years, a series of aptamer-targeted chemotherapeutic models have been successfully established and further assessed in preclinical studies. Aptamers can be directly combined with drugs through chemical bonding or physical mixture to form a simple drug delivery system [106]. To broaden the clinical application of aptamer-drug conjugates, a new effective strategy should develop the therapeutic aptamer-based nanomedicines by conjugating aptamers with nanomaterials, which may improve the drug load and biological stability, while reducing the renal clearance rate to prolong the half-life $[107,108]$. To enhance the stability of aptamers, Leach et al. [20] connected a small single-stranded DNA to
RNA aptamer A10-3-J1 which can specifically recognize PSMA, to constitute DNA-RNA hybrid aptamer. Then, using superparamagnetic iron oxide nanoparticles (SPION) as carriers, doxorubicin (DOX) was loaded onto the aptamer-coupled SPIONs to form a targeted drug delivery system. Several tests confirmed that the newly constructed DNA-RNA hybrid aptamer did not change the high affinity of the original RNA aptamer A10-3-J1 to PSMA. The hybrid aptamer-mediated delivery platform of targeted drug (APTSPIO-DOX) could enhance the cytotoxicity of target cells while minimizing the damage to nontarget cells. Chen et al. [109] prepared a carboxy-terminated poly (D, L-lactide-co-glycolide)-block-poly(ethylene glycol) (PLGA-bPEG-COOH) diblock copolymer by a solvent diffusion method, and the chemotherapy drug docetaxel (DTX) was encapsulated to it. Subsequently, the anti-PSMA aptamer was coated to drug-loaded nanoparticles (DTX-NPs) by EDC/NHS coupling technology. These aptamer-based DTX-APT-NPs are expected to specifically deliver DTX to $\mathrm{PSMA}^{+}$prostate cancer and to enhance the antitumor effect of DTX through aptamer-mediated intracellular delivery. 
6.2. Gene Therapy Mediated by Aptamer Functionalized Nanocomposites. Gene therapy plays a significant role in tumor treatment, and its key-point is to identify a suitable carrier to effectively introduce nucleic acid drug. The combination of aptamers with nanomaterials can provide multiple application advantages for gene drugs, such as tumortargeting ability, and enhance transfection efficiency by receptor-mediated internalization pathways [110]. CRISPR/ Cas9 has been developed as a new type of nucleic acid drugs to treat various diseases for its powerful ability to suppress the expression of target genes [111]. Similar to other biological medicines, CRISPR/Cas9-based treatment often requires a carrier for delivery to the target tissue or cell. Zhen et al. [51] connected cationic liposomes to the A10 aptamer using the postinsertion method to efficiently and flexibly introduce therapeutic CRISPR/Cas9 into prostate cancer cells, binding it to the target gene Polo-like kinase 1 (PLK-1). PLK-1 is a prosurvival gene that is overexpressed in most tumors, which could be acted as a valid target since its antiapoptosis effect, thus, knocking out the PLK-1 was believed to reduce the viability of prostate cancer cell. Results from in vitro and in vivo assays have shown that A10 aptamer-mediated chimera can specifically bind to prostate cancer cells with high PSMA expression, which can yield to significant effects of gene silencing, and subsequently significant tumor recession.

\subsection{Thermotherapy Mediated by Aptamer Functionalized} Nanocomposites. Thermotherapy has become the fifth largest tumor treatment after radiotherapy, chemotherapy, surgery, and immunotherapy, which aims to cause irreversible changes in tumor cells and further lead to their apoptosis by thermal energy at a certain temperature [112]. Many different types of energy sources, including radiofrequency, microwave, ultrasound, and laser irradiation, have been used for the external delivery of thermal energy [113]. The tumor tissue with rapid angiogenesis has an abundant blood supply, which may result in incomplete structure of the capillary wall. The disordered branches and distorted structure of the capillaries are easy to rupture, with the addition of the circulation blocking caused by tumor compression, causing a consequence that cancer cells are more sensitive to heat than normal cells. It is generally recognized that $42-44^{\circ} \mathrm{C}$ is the effective temperature for tumor treatment, and there is no damage to normal tissue [114]. As a relatively noninvasive cancer treatment, hyperthermia is usually applied as an adjunct to an already established treatment modality, especially radiotherapy and chemotherapy [115]. How to uniformly heat up the tumor tissue to the required temperature for treatment without damaging normal tissue is a major technical problem in hyperthermia. Aptamer functionalized nanocomposites have the features of binding with specific target substances on the surface of tumor cells, thus, applying this to tumor hyperthermia could effectively decrease the thermal damage of normal tissues, further leading to tumor hyperthermia developing towards precise positioning. Magnetic induction hyperthermia (MIH) based on magnetic nanomaterials is one of the newly developed antitumor therapies in recent years, which is recognized as a "green treatment." The magnetic medium is distributed to the treatment volume through implantation or intervention, and under the action of alternating magnetic field (AMF), the magnetic medium generates high-frequency vibration and a large amount of heat due to the Néelian relaxation mechanism and/or Brownian motion, so that the tumor tissue can reach and maintain the required temperature for effective treatment, thereby inducing necrosis and apoptosis of tumor cells [116]. Professor Johannsen et al. from Humboldt University in Germany [117] were considered as the pioneers to conduct clinical trials of magnetic induction heating therapy in prostate cancer, and they proved the heating technique using magnetic nanoparticles was feasible and well tolerated in patients with locally recurrent prostate cancer. Moreover, Johannsen and his team further evaluated the effects of thermotherapy mediated by magnetic fluid in combination with external radiation in the orthotopic rat model of prostate cancer. They achieved relatively good efficacy and verified a synergism between these two modalities [118]. Guo et al. [53] constructed MUC-1 aptamermediated nanoiron particles (APT-NPs), followed by exploration of using APT-NPs as magnetic medium for targeted magnetic hyperthermia in tumor. In vitro cell assay showed that APT-NPs could significantly increase the thermal damage of MUC1-positive MCF-7 cells under the AMF, but had no obvious thermal damage in MUC1-negative HepG2 cells, suggesting that MUC1 aptamer-modified magnetic nanoparticles have potential application prospects in targeted hyperthermia of adenocarcinoma, including prostate cancer. Photothermal therapy (PTT) is a technique which uses photothermal conversion materials to generate heat under near-infrared (NIR) laser radiation to kill tumor cells. The aptamer-gold nanorod (AuNR) conjugate [57] showed good longitudinal plasmon resonance absorption in the NIR range and can effectively transfer NIR light to local area for heating, which has been used in PTT for leukemia. Wang et al. [58] screened an aptamer (CSC1) specifically targeting DU145 prostate cancer cells and another targeting tumor stem cells (CSC13), then modified them on the surface of AuNRs. Through the covalent connection between aptamer and AuNRs, specific cell targeting and selective PTT were achieved under the NIR irradiation.

\subsection{Combination Therapy Mediated by Aptamer} Functionalized Nanocomposites. For some intractable tumors, two or more drugs or combination therapy of different means are often considered to improve curative effect or exert synergistic effects, namely, obtain the result of " $1+1>2$." For example, a combination of treatment modalities such as surgery, radiation therapy, photodynamic therapy, gene therapy, and/or chemotherapy is widely considered to achieve synergistic therapeutic efficacy [119, 120] (Figure 6).

Combination of chemotherapeutic drugs that have different mechanisms of action or different efficacies which have additive or synergistic effects on overcoming drug resistance. Furthermore, it also allows the use of lower doses and can reduce intolerable side effects [121]. Zhang et al. [122] established nanodrug codelivery system by using the A10 RNA aptamer with multiple CG sequences as a target 


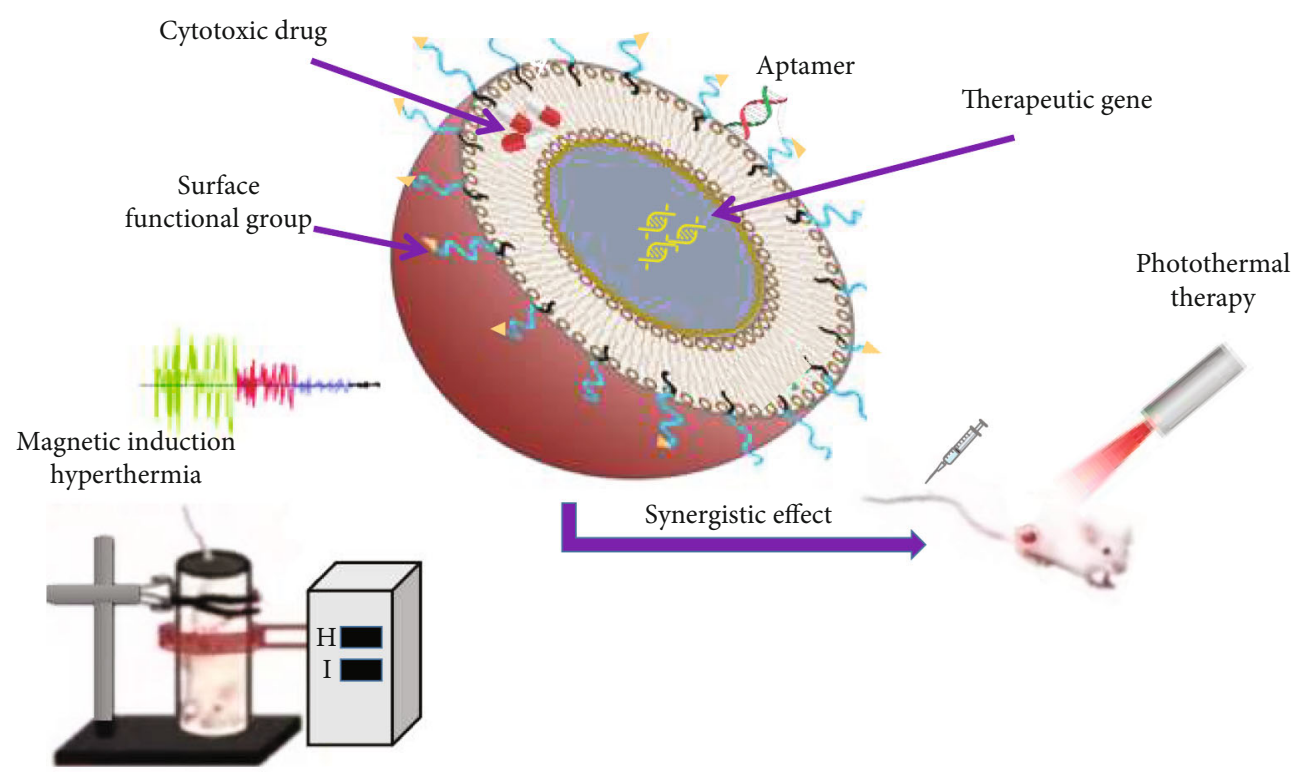

FIgURE 6: Schematic illustration of integrated therapeutic platforms based on aptamer functionalized nanocomposites. Nanoparticles have been widely used in drug and gene delivery. Among them, magnetic nanoparticles are also useful for thermal therapy, which involves localized heating produced by coupling these particles to an ac magnetic field. Meanwhile, gold nanospheres, nanorods have be extensively used in photothermal therapy. A combination of treatment modalities above is generally considered to achieve synergistic therapeutic efficacy.

molecule and PLGA-b-PEG block copolymer as a drug carrier, which can simultaneously deliver both hydrophilic DOX and hydrophobic DTX, for targeted synergistic therapy of prostate cancer. The drug codelivery system consisting of nanoparticle-aptamer bioconjugates could specifically be targeted to PCa cells with PSMA overexpression and showed a significantly higher tumor killing activity than the single drug. Chemotherapy combined with gene therapy can kill cancer cells systemically, including cancer cells that have spread to distant sites [123]. Research shows that siRNA-mediated silencing of Bcl-2, an antiapoptotic gene, synergized with even small amounts of DOX was successful in inducing cancer cell death [124]. Kim et al. [49] grafted polyethyleneimine (PEI) to PEG as a vehicle for shRNAs against Bcl-xL delivery, and then its surface was further conjugated with anti-PSMA aptamer A10. After the intercalation of DOX, the shRNA/PEI-PEG-APT/DOX conjugates (APs) were finally obtained. In vitro cell culture experiments showed that the nanoplatform efficiently and selectively codelivered both Bcl-xL-specific shRNA and DOX to LNCaP cells through aptamer-mediated binding to PSMA which is expressed on the cell surfaces. Furthermore, the APs showed impressive synergistic effect and remarkably increased cytotoxicity in target cells, providing another option for prostate cancer treatment. Thermotherapy was often exploited as a combinatorial approach to enhance the sensitivity of traditional cancer therapies such as radiotherapy, chemotherapy, immunotherapy, and gene therapy. Such a combination therapy has been rapidly realized as a strategy to improve the tumor treatment efficacy, which greatly satisfies the growing demand of personalized medicine and oncology development [125]. Zhao et al. [126] loaded DTX into thermosensitive liposomes coated with gold nanoshells, then coupled it with AS1411 aptamers, finally successfully constructed a aptamer-targeted nanoparticle drug carrier with near-infrared photothermal response. The drug delivery system showed light-thermal sensitivity, which achieved spatiotemporally controlled drug release under $808 \mathrm{~nm}$ laser irradiation and the acidic environment of tumor, and improved DTX accumulation in tumor tissues by cell internalization. In addition to using as a targeting agent, in this study, the AS1411 aptamer [127] is introduced as a biotherapeutic agent combined with chemotherapy. Finally, the experiments conducted in vitro and in vivo demonstrated the nanosystem can synergistically inhibit tumor growth by the combination of chemotherapy, photothermal therapy, and biological therapy. This research greatly contributes to the use of nanocarrier-based delivery of different therapeutic modalities for the synergistic treatment of cancer.

\section{Aptamer Functionalized Nanocomposite Mediated Integration of Diagnosis and Treatment}

The same type of tumor occurring in different individuals shows diverse invasiveness, sensitivity to anticancer drugs, progression, and prognosis due to the multiple comprehensive factors, such as genetic and environmental factors $[128,129]$. With the in-depth study in the biological basis of $\mathrm{PCa}$ and its malignant proliferation, metastasis, and tumor escape, multiple potential targets, such as androgen receptors, signaling pathways, and tumor-associated antigens, have been gradually unveiled $[130,131]$, coupled with 
the continuous breakthrough in the field of aptamer technology and nanomedicine, the increasing explorations on the "integration of diagnosis and treatment" of prostate cancer are under way, achieving initial outcomes. While performing in situ diagnosis of prostate cancer by different detection methods, dynamically monitoring its curative effect, then adjusting and optimizing the therapeutic strategies in time, so as to work out the corresponding therapeutic plans according to the specific conditions of different patients, which is widely concerned in the era of emphasizing individualized precision medicine $[132,133]$. Wang et al. [52] constructed a novel multifunctional A10 aptamer-modified TCL-SPION and then took advantage of SPIO's unique superparamagnetism and aptamers' active targeting for PCa molecular imaging and therapy. The TCL-SPIONAPT bioconjugates could be acted as therapeutic carriers for selectively delivering DOX to PSMA-expressing prostate cancer cells. Meanwhile, the potential of bioconjugates as targeted MRI contrast agents was verified. Kim et al. [56] coated the DOX-loaded A9 aptamer to gold nanoparticles (GNPs), forming a multifunctional nanoplatform for monitoring and killing prostate cancer. As a targeted molecular computed tomography (CT) imaging system, the constructed aptamer-conjugated GNP was capable of specific imaging for prostate cancer cells with overexpressed PSMA, which showed more than 4-fold greater CT intensity for a targeted LNCaP cell than that of nontargeted PC-3 cells. Apart from that, the nanocomposite improved some defects of traditional CT iodinated contrast agents, such as lack of targeting, short imaging time, and nephrotoxicity. Furthermore, as the anticancer drug delivery vehicle, aptamermediated GNPs after loading of DOX were significantly more potent against targeted cells. In a word, the PSMA aptamer described above served the dual functions of escort molecule and drug delivery carrier, achieving the integration of diagnoses and treatment for prostate cancer. Lin et al. [55] coupled the NIR-CuInS2 QDs with MUC-1 aptamer, followed by insertion of daunorubicin (DNR) into the double-stranded CG sequence of MUC1-QDs to form DNR-MUC1-QDs complexes. The aptamer-functionalized bio-nanosystem can not only deliver DNR to the targeted prostate cancer cells but also detect DNR by changing the photoluminescence intensity of CuInS2QDs, while rendering NIR imaging of cancer cells. As a novel bionanomedicine sensing and delivery system, the constructed DNR-MUC1-QDs can integrate tumor cell imaging and targeted therapy together, with high specificity and sensitivity. $\mathrm{Wu}$ et al. [48] developed A10-3.2 aptamer-modified poly (lactide-co-glycolide) (PLGA) nanobubbles as ultrasound contrast agents and drug carriers. The ultrasonic radiation of tumors can stimulate drug release and temporarily improve the permeability of cell membranes, thereby enhancing the diffusion of chemotherapeutics in the tumor mass and decreasing drug concentration gradients. After encapsulating paclitaxel (PTX), the theranostic nanoplatform based on PTX-A10-3.2-PLGA achieved the delivery of anticancer drugs, while obtaining ultrasound images for prostate cancer. Additionally, it also provided lowfrequency ultrasound-triggered treatment for prostate can- cer, effectively combining "PTX chemotherapy, ultrasound imaging and tumor cell-targeting," which provides a strategy for both prostate cancer imaging and therapy.

\section{Deficiencies and Prospects}

Aptamer functionalized nanocomposites have exhibited multiple advantages, and great progresses have been made in their basic research and clinical application in the fields of targeted drug delivery, diagnosis, and treatment of tumor. Among them, aptamers targeting PCa have also displayed superiorities, such as high targeting ability and high specificity in relevant research on PCa. However, due to the high production costs of large-scale aptamer synthesis and the uncertain effect in human body, most research is still limited to experimental study, and there is still a long way to go to clinical application. Nevertheless, it is believed that with the continuous progress of medical technology, more and more excellent aptamers will be screened and synthesized, the research concerning aptamer functionalized nanocomposites in PCa will be further intensified and improved, and large scale in vivo experiments and clinical trials are expected to be conducted in the near future, so as to pave the way for the clinical application of nanocomposites in PCa.

\section{Abbreviations}

$\begin{array}{ll}\text { PCa: } & \text { Prostate cancer } \\ \text { SELEX: } & \text { Systematic evolution of ligands by exponen- } \\ & \text { tial enrichment } \\ \text { PSMA: } & \text { Prostate-specific membrane antigen } \\ \text { PSA: } & \text { Prostate-specific antigen } \\ \text { BPH: } & \text { Benign prostatic hyperplasia } \\ \text { AR: } & \text { Androgen receptor } \\ \text { 6-MCH: } & \text { 6-Mercapto-1-hexanol } \\ \text { OCP: } & \text { Open circuit potential } \\ \text { EATR: } & \text { Enzyme-assisted target recycling } \\ \text { HRE: } & \text { Hormone response element } \\ \text { CRPC: } & \text { Castration-resistant prostate cancer } \\ \text { EPR: } & \text { Enhanced permeability and retention } \\ \text { QDs: } & \text { Quantum dots } \\ \text { VEGF: } & \text { Vascular endothelial growth factor } \\ \text { CEA: } & \text { Carcinoembryonic antigen } \\ \text { EpCAM: } & \text { Epidermal adhesion molecule } \\ \text { FRET: } & \text { Fluorescence resonance energy transfer } \\ \text { IFE: } & \text { Internal filter effect } \\ \text { MSNs: } & \text { Mesoporous silica nanoparticles } \\ \text { MRI: } & \text { Magnetic resonance imaging } \\ \text { GBCAs: } & \text { Gadolinium- (III-) based contrast agents } \\ \text { TCL-SPIONs: } & \text { Superparamagnetic iron oxide nanoparticles } \\ \text { UCAs: } & \text { Ultrasound contrast agents } \\ \text { DRE: } & \text { Digital rectal examination } \\ \text { MWCNTs: } & \text { Multiwalled carbon nanotubes } \\ \text { PEG: } & \text { Poly(ethylene glycol) } \\ \text { PET: } & \text { Positron emission tomography } \\ \text { SPECT: } & \text { Single photon emission computed } \\ \text { mCRPC: } & \text { tomography } \\ & \end{array}$

mCRPC: 
Metastatic castration-resistant prostate cancer

$\begin{array}{ll}\text { SPION: } & \text { Superparamagnetic iron oxide nanoparticles } \\ \text { DOX: } & \text { Doxorubicin } \\ \text { DTX: } & \text { Docetaxel } \\ \text { EDC/NHS: } & \begin{array}{l}\text { 1-Ethyl-3-(3-dimethylaminopropyl)carbo- } \\ \text { diimide/N-hydroxy-succinamide }\end{array} \\ \text { PLK-1: } & \text { Polo-like kinase 1 } \\ \text { MIH: } & \text { Magnetic induced hyperthermia } \\ \text { AuNR: } & \text { Gold nanorod } \\ \text { AMF: } & \text { Alternating magnetic field } \\ \text { PTT: } & \text { Photothermal therapy } \\ \text { NIR: } & \text { Near-infrared } \\ \text { PEI: } & \text { Polyethyleneimine } \\ \text { GNPs: } & \text { Gold nanoparticles } \\ \text { CT: } & \text { Computed tomography } \\ \text { DNR: } & \text { Daunorubicin } \\ \text { PLGA: } & \text { Poly(lactide-co-glycolide) } \\ \text { PTX: } & \text { Paclitaxel. }\end{array}$

\section{Consent}

All authors read and approved the final manuscript.

\section{Conflicts of Interest}

The authors declare that they have no competing interests.

\section{Authors' Contributions}

Xiaoqian Feng conceived and drafted the manuscript; Yinxing Zhu collected partial references and contributed to the writing; Fujin Wang, Ting Guo, and Xiaofeng Dou participated in the analysis and collation of literature; Mei Lin designed this review study and gave constructive guidance and made critical revisions; Weizhong Tian gave some guidance and contributed to the writing. The authors read and approved the final manuscript.

\section{Acknowledgments}

We thank the members in Medical Imaging Department and Institute of Clinical Medicine of Taizhou People's Hospital Affiliated to Nantong University for contributions. The work is financially supported by the National Natural Science Foundation of China (81571797), the Social Development Plan of Taizhou, China (TS202004), the Natural Science Foundation of Nanjing University of Chinese Medicine, China (XZR2020093), and Taizhou People's Hospital Medical Innovation Team Foundation, China (CXTDA201901).

\section{References}

[1] R. L. Siegel, K. D. Miller, and A. Jemal, "Cancer statistics, 2019," CA: A Cancer Journal for Clinicians, vol. 69, no. 1, pp. 7-34, 2019.

[2] A. Lemanska, D. P. Dearnaley, R. Jena, M. R. Sydes, and S. Faithfull, "Older age, early symptoms and physical function are associated with the severity of late symptom clusters for men undergoing radiotherapy for prostate cancer," Clinical Oncology, vol. 30, no. 6, pp. 334-345, 2018.

[3] J. H. Yan, H. J. Xiong, S. D. Cai et al., "Advances in aptamer screening technologies," Talanta, vol. 200, pp. 124-144, 2019.

[4] J. Xie, Y. Zhang, C. Y. Yan et al., "High-performance PEGylated $\mathrm{Mn}-\mathrm{Zn}$ ferrite nanocrystals as a passive-targeted agent for magnetically induced cancer theranostics," Biomaterials, vol. 35, no. 33, pp. 9126-9136, 2014.

[5] G. Y. Chen, I. Roy, C. H. Yang, and P. N. Prasad, "Nanochemistry and nanomedicine for nanoparticle-based diagnostics and therapy," Chemical Reviews, vol. 116, no. 5, pp. 2826-2885, 2016.

[6] A. D. Ellington and J. W. Szostak, "In vitro_selection of RNA molecules that bind specific ligands," Nature, vol. 346, no. 6287, pp. 818-822, 1990.

[7] C. Tuerk and L. Gold, "Systematic evolution of ligands by exponential enrichment: RNA ligands to bacteriophage T4 DNA polymerase," Science, vol. 249, no. 4968, pp. 505-510, 1990.

[8] Y. Morita, M. Leslie, H. Kameyama, D. E. Volk, and T. Tanaka, "Aptamer therapeutics in cancer: current and future," Cancers, vol. 10, no. 3, p. 80, 2018.

[9] M. H. Ali, M. E. Elsherbiny, and M. Emara, "Updates on aptamer research," International Journal of Molecular Sciences, vol. 20, no. 10, p. 2511, 2019.

[10] Y. Zhang, B. S. Lai, and M. Juhas, "Recent advances in aptamer discovery and applications," Molecules, vol. 24, no. 5, p. 941, 2019.

[11] Z. J. Zhuo, Y. Y. Yu, M. L. Wang et al., "Recent advances in SELEX technology and aptamer applications in biomedicine," International Journal of Molecular Sciences, vol. 18, no. 10, p. 2142, 2017.

[12] L. L. Dong, Q. W. Tan, W. Ye, et al., "Screening and identifying a novel ssDNA aptamer against alpha-fetoprotein using CE-SELEX," Scientific Reports, vol. 5, no. 1, p. 15552, 2015.

[13] M. Srinivasarao, C. V. Galliford, and P. S. Low, "Principles in the design of ligand-targeted cancer therapeutics and imaging agents," Nature Reviews. Drug Discovery, vol. 14, no. 3, pp. 203-219, 2015.

[14] E. Campos-Fernández, N. Oliveira Alqualo, L. C. Moura Garcia et al., "The use of aptamers in prostate cancer: a systematic review of theranostic applications," Clinical Biochemistry, vol. 93, pp. 9-25, 2021.

[15] X. J. Xu, D. D. Dickey, S.-J. Chen, and P. H. Giangrande, "Structural computational modeling of RNA aptamers," Methods, vol. 103, pp. 175-179, 2016.

[16] O. Boyacioglu, C. H. Stuart, G. Kulik, and W. H. Gmeiner, "Dimeric DNA aptamer complexes for high-capacitytargeted drug delivery using $\mathrm{pH}$-sensitive covalent linkages," Molecular Therapy - Nucleic Acids, vol. 2, no. 7, article e107, 2013.

[17] S. E. Lupold, B. J. Hicke, Y. Lin, and D. S. Coffey, "Identification and characterization of nuclease-stabilized RNA molecules that bind human prostate cancer cells via the prostatespecific membrane antigen," Cancer Research, vol. 62, no. 14, pp. 4029-4033, 2002.

[18] W. M. Rockey, F. J. Hernandez, S. Y. Huang et al., "Rational truncation of an RNA aptamer to prostate-specific membrane antigen using computational structural modeling," Nucleic Acid Therapeutics, vol. 21, no. 5, pp. 299-314, 2011. 
[19] J. P. Dassie, X. Y. Liu, G. S. Thomas et al., "Systemic administration of optimized aptamer-siRNA chimeras promotes regression of PSMA-expressing tumors," Nature Biotechnology, vol. 27, no. 9, pp. 839-846, 2009.

[20] J. C. Leach, A. Wang, K. Ye, and S. Jin, “A RNA-DNA hybrid aptamer for nanoparticle-based prostate tumor targeted drug delivery," International Journal of Molecular Sciences, vol. 17, no. 3, p. 380, 2016.

[21] Y. Wang, Y. Luo, T. Bing et al., "DNA aptamer evolved by cell-SELEX for recognition of prostate cancer," PLoS One, vol. 9, no. 6, article e100243, 2014.

[22] M. L. Duan, Y. Q. Long, C. Yang et al., "Selection and characterization of DNA aptamer for metastatic prostate cancer recognition and tissue imaging," Oncotarget, vol. 7, no. 24, pp. 36436-36446, 2016.

[23] S. Kouhpayeh, A. R. Einizadeh, Z. Hejazi et al., "Antiproliferative effect of a synthetic aptamer mimicking androgen response elements in the LNCaP cell line," Cancer Gene Ther$a p y$, vol. 23, no. 8, pp. 254-257, 2016.

[24] J. Wang, K. Sefah, M. B. Altman et al., "Aptamer-conjugated nanorods for targeted photothermal therapy of prostate cancer stem cells," Chemistry - An Asian Journal, vol. 8, no. 10, pp. 2417-2422, 2013.

[25] B. P. Gray, L. Kelly, D. P. Ahrens et al., "Tunable cytotoxic aptamer-drug conjugates for the treatment of prostate cancer," Proceedings of the National Academy of Sciences, vol. 115, no. 18, pp. 4761-4766, 2018.

[26] N. Savory, K. Abe, K. Sode, and K. Ikebukuro, "Selection of DNA aptamer against prostate specific antigen using a genetic algorithm and application to sensing," Biosensors \& Bioelectronics, vol. 26, no. 4, pp. 1386-1391, 2010.

[27] A. E. Karpik, B. P. Crulhas, C. B. Rodrigues, G. R. Castro, and V. A. Pedrosa, "Aptamer-based biosensor developed to monitor MUC1 released by prostate cancer cells," Electroanalysis, vol. 29, no. 10, pp. 2246-2253, 2017.

[28] P. J. Bates, D. A. Laber, D. M. Miller, S. D. Thomas, and J. O. Trent, "Discovery and development of the G-rich oligonucleotide AS1411 as a novel treatment for cancer," Experimental and Molecular Pathology, vol. 86, no. 3, pp. 151-164, 2009.

[29] A. Ghosh and W. D. Heston, "Tumor target prostate specific membrane antigen (PSMA) and its regulation in prostate cancer," Journal of Cellular Biochemistry, vol. 91, no. 3, pp. 528-539, 2004.

[30] S. R. Denmeade, A. M. Mhaka, D. M. Rosen et al., "Engineering a prostate-specific membrane antigen-activated tumor endothelial cell prodrug for cancer therapy," Science Translational Medicine, vol. 4, no. 140, 2012.

[31] J. G. Zhao, S. P. Wang, S. B. Zhang et al., "Peptide cleavagemediated photoelectrochemical signal on-off via CuS electronic extinguisher for PSA detection," Biosensors \& Bioelectronics, vol. 150, article 111958, 2020.

[32] A. Diaz-Fernandez, R. Miranda-Castro, N. de-Los-SantosAlvarez, M. J. Lobo-Castanon, and P. Estrela, "Impedimetric aptamer-based glycan PSA score for discrimination of prostate cancer from other prostate diseases," Biosensors and Bioelectronics, vol. 175, article 112872, 2021.

[33] V. K. Tamboli, N. Bhalla, P. Jolly et al., "Hybrid synthetic receptors on MOSFET devices for detection of prostate specific antigen in human plasma," Analytical Chemistry, vol. 88, no. 23, pp. 11486-11490, 2016.
[34] L. C. C. Wong, P. Jolly, and P. Estrela, "Development of a sensitive multiplexed open circuit potential system for the detection of prostate cancer biomarkers," BioNanoScience, vol. 8, no. 2, pp. 701-706, 2018.

[35] M. J. Chen, Z. W. Tang, and C. B. Ma, “A fluorometric aptamer based assay for prostate specific antigen based on enzyme-assisted target recycling," Sensors and Actuators B: Chemical, vol. 302, article 127178, 2020.

[36] K. E. Knudsen and H. I. Scher, "Starving the addiction: new opportunities for durable suppression of AR signaling in prostate cancer," Clinical Cancer Research, vol. 15, no. 15, pp. 4792-4798, 2009.

[37] A. Drabik, J. Ner-Kluza, P. Mielczarek, L. Civit, G. Mayer, and J. Silberring, "Advances in the study of aptamer-protein target identification using the chromatographic approach," Journal of Proteome Research, vol. 17, no. 6, pp. 2174-2181, 2018.

[38] N. Wong, P. Major, A. Kapoor, and F. X. Wei, “Amplification of MUC1 in prostate cancer metastasis and CRPC development," Oncotarget, vol. 7, no. 50, pp. 83115-83133, 2016.

[39] S. M. Taghdisi, N. M. Danesh, M. Ramezani, R. YazdianRobati, and K. Abnous, "A novel AS1411 aptamer-based three-way junction pocket DNA nanostructure loaded with doxorubicin for targeting cancer cells in vitro and in vivo," Molecular Pharmaceutics, vol. 15, no. 5, pp. 1972-1978, 2018.

[40] S. Zununi Vahed, N. Fathi, M. Samiei, S. Maleki Dizaj, and S. Sharifi, "Targeted cancer drug delivery with aptamerfunctionalized polymeric nanoparticles," Journal of Drug Targeting, vol. 27, no. 3, pp. 292-299, 2019.

[41] B. L. Santini, M. Zúñiga-Bustos, A. Vidal-Limon, J. B. Alderete, S. A. Águila, and V. A. Jiménez, "In SilicoDesign of novel mutant Anti-MUC1 aptamers for targeted cancer therapy," Journal of Chemical Information and Modeling, vol. 60, no. 2, pp. 786-793, 2020.

[42] Y. Y. Yu, C. Liang, Q. X. Lv et al., "Molecular selection, modification and development of therapeutic oligonucleotide aptamers," International Journal of Molecular Sciences, vol. 17, no. 3, p. 358, 2016.

[43] R. Li, K. Zheng, C. Yuan, Z. Chen, and M. D. Huang, "Be active or not: the relative contribution of active and passive tumor targeting of nanomaterials," Nanotheranostics, vol. 1, no. 4, pp. 346-357, 2017.

[44] M. Omer, V. L. Andersen, J. S. Nielsen, J. Wengel, and J. Kjems, "Improved cancer targeting by multimerizing aptamers on nanoscaffolds," Molecular Therapy - Nucleic Acids, vol. 22, pp. 994-1003, 2020.

[45] F. F. Gu, C. L. Hu, Q. M. Xia, C. N. Gong, S. Gao, and Z. J. Chen, "Aptamer-conjugated multi-walled carbon nanotubes as a new targeted ultrasound contrast agent for the diagnosis of prostate cancer," Journal of Nanoparticle Research, vol. 20, no. 11, p. 303, 2018.

[46] H. J. Zhang, L. Hou, X. J. Jiao et al., "In vitro and in vivo evaluation of antitumor drug-loaded aptamer targeted singlewalled carbon nanotubes system," Current Pharmaceutical Biotechnology, vol. 14, no. 13, pp. 1105-1117, 2014.

[47] Z. Y. Deng, Q. X. Yang, Y. B. Peng et al., "Polymeric Engineering of Aptamer-Drug Conjugates for Targeted Cancer Therapy," Bioconjugate Chemistry, vol. 31, no. 1, pp. 37-42, 2019.

[48] M. Wu, Y. Wang, Y. R. Wang et al., "Paclitaxel-loaded and A10-3.2 aptamer-targeted poly(lactide-co-glycolic acid) 
nanobubbles for ultrasound imaging and therapy of prostate cancer," International Journal of Nanomedicine, vol. Volume 12, pp. 5313-5330, 2017.

[49] E. Kim, Y. Jung, H. Choi et al., "Prostate cancer cell death produced by the co-delivery of Bcl-xL shRNA and doxorubicin using an aptamer-conjugated polyplex," Biomaterials, vol. 31, no. 16, pp. 4592-4599, 2010.

[50] X. Z. Fan, Y. L. Guo, L. F. Wang, X. Y. Xiong, L. H. Zhu, and K. J. Fang, "Diagnosis of prostate cancer using anti-PSMA aptamer A10-3.2-oriented lipid nanobubbles," International Journal of Nanomedicine, vol. Volume 11, pp. 3939-3950, 2016.

[51] S. Zhen, Y. Takahashi, S. Narita, Y. C. Yang, and X. Li, “Targeted delivery of CRISPR/Cas9 to prostate cancer by modified gRNA using a flexible aptamer-cationic liposome," Oncotarget, vol. 8, no. 6, pp. 9375-9387, 2017.

[52] A. Z. Wang, V. Bagalkot, C. C. Vasilliou et al., "Superparamagnetic iron oxide nanoparticle-aptamer bioconjugates for combined prostate cancer imaging and therapy," ChemMedChem, vol. 3, no. 9, pp. 1311-1315, 2008.

[53] F. Q. Guo, Y. Hu, L. Y. Yu et al., "Enhancement of thermal damage to adenocarcinoma cells by iron nanoparticles modified with MUC1 aptamer," Journal of Nanoscience and Nanotechnology, vol. 16, no. 3, pp. 2246-2253, 2016.

[54] W. Argoubi, A. Sánchez, C. Parrado, N. Raouafi, and R. Villalonga, "Label-free electrochemical aptasensing platform based on mesoporous silica thin film for the detection of prostate specific antigen," Sensors and Actuators B: Chemical, vol. 255, pp. 309-315, 2018.

[55] Z. H. Lin, Q. Ma, X. F. Fei, H. Zhang, and X. G. Su, “A novel aptamer functionalized $\mathrm{CuInS}_{2}$ quantum dots probe for daunorubicin sensing and near infrared imaging of prostate cancer cells," Analytica Chimica Acta, vol. 818, pp. 54-60, 2014.

[56] D. Kim, Y. Y. Jeong, and S. Jon, “A drug-loaded aptamer-gold nanoparticle bioconjugate for combined CT imaging and therapy of prostate cancer," ACS Nano, vol. 4, no. 7, pp. 3689-3696, 2010.

[57] Y. F. Huang, K. Sefah, S. Bamrungsap, and H. T. Chang, "Selective photothermal therapy for mixed cancer cells using aptamer-conjugated nanorods," Langmuir, vol. 24, no. 20, pp. 11860-11865, 2008.

[58] Z. P. Guan, T. S. Zhang, H. Zhu et al., "Simultaneous Imaging and Selective Photothermal Therapy through AptamerDriven Au Nanosphere Clustering," The Journal of Physical Chemistry Letters, vol. 10, no. 2, pp. 183-188, 2018.

[59] N. A. Correia, L. T. A. Batista, R. J. M. Nascimento et al., "Detection of prostate cancer by Raman spectroscopy: a multivariate study on patients with normal and altered PSA values," Journal of Photochemistry and Photobiology. B, vol. 204, p. 111801, 2020.

[60] M. Negahdary, N. Sattarahmady, and H. Heli, "Advances in prostate specific antigen biosensors-impact of nanotechnology," Clinica Chimica Acta, vol. 504, pp. 43-55, 2020.

[61] L. L. Shi, L. Ba, Y. Xiong, and G. Peng, "A hybridization chain reaction based assay for fluorometric determination of exosomes using magnetic nanoparticles and both aptamers and antibody as recognition elements," Microchimica Acta, vol. 186, no. 12, article 796, 2019.

[62] F. Tan, Y. Yang, X. X. Xie et al., "Prompting peroxidase-like activity of gold nanorod composites by localized surface plasmon resonance for fast colorimetric detection of prostate specific antigen," Analyst, vol. 143, no. 20, pp. 5038-5045, 2018.
[63] C. Ibau, M. K. Md Arshad, S. C. B. Gopinath, M. N. M. Nuzaihan, M. F. M. Fathil, and P. Estrela, "Gold interdigitated triple-microelectrodes for label-free prognosticative aptasensing of prostate cancer biomarker in serum," Biosensors and Bioelectronics, vol. 136, pp. 118-127, 2019.

[64] T. Y. Wang, E. Viennois, D. Merlin, and G. L. Wang, "Microelectrode miRNA sensors enabled by enzymeless electrochemical signal amplification," Analytical Chemistry, vol. 87, no. 16, pp. 8173-8180, 2015.

[65] G. Perry, F. Cortezon-Tamarit, and S. I. Pascu, "Detection and monitoring prostate specific antigen using nanotechnology approaches to biosensing," Frontiers of Chemical Science and Engineering, vol. 14, no. 1, pp. 4-18, 2020.

[66] X. Y. Gao, L. Qi, K. Liu, C. C. Meng, Y. C. Li, and H. Z. Yu, "Exonuclease I-assisted general strategy to convert aptamerbased electrochemical biosensors from "signal-off" to "signal-on"," Analytical Chemistry, vol. 92, no. 9, pp. 62296234, 2020.

[67] B. Kavosi, A. Salimi, R. Hallaj, and F. Moradi, "Ultrasensitive electrochemical immunosensor for PSA biomarker detection in prostate cancer cells using gold nanoparticles/PAMAM dendrimer loaded with enzyme linked aptamer as integrated triple signal amplification strategy," Biosensors and Bioelectronics, vol. 74, pp. 915-923, 2015.

[68] B. Kavosi, A. Navaee, and A. Salimi, "Amplified fluorescence resonance energy transfer sensing of prostate specific antigen based on aggregation of CdTe QDs/antibody and aptamer decorated of AuNPs-PAMAM dendrimer," Journal of Luminescence, vol. 204, pp. 368-374, 2018.

[69] S. Mehmood, A. Z. Khan, M. Bilal, A. Sohail, and H. M. N. Iqbal, "Aptamer-based biosensors: a novel toolkit for early diagnosis of cancer," Materials Today Chemistry, vol. 12, pp. 353-360, 2019.

[70] Y. Yang, G. B. Hu, W. B. Liang et al., "An AIEgen-based 2D ultrathin metal-organic layer as an electrochemiluminescence platform for ultrasensitive biosensing of carcinoembryonic antigen," Nanoscale, vol. 12, no. 10, pp. 5932-5941, 2020.

[71] S. de Wit, M. Manicone, E. Rossi et al., "EpCAM ${ }^{\text {high }}$ and EpCAM $^{\text {low }}$ circulating tumor cells in metastatic prostate and breast cancer patients," Oncotarget, vol. 9, no. 86, pp. 35705-35716, 2018.

[72] L. H. Pan, S. H. Kuo, T. Y. Lin, C. W. Lin, P. Y. Fang, and H. W. Yang, "An electrochemical biosensor to simultaneously detect VEGF and PSA for early prostate cancer diagnosis based on graphene oxide/ssDNA/PLLA nanoparticles," Biosensors and Bioelectronics, vol. 89, Part 1, pp. 598-605, 2017.

[73] J. Y. Chong, H. Y. Chong, and J. H. Lee, "A chemiluminescent dual-aptasensor capable of simultaneously quantifying prostate specific antigen and vascular endothelial growth factor," Analytical Biochemistry, vol. 564-565, pp. 102-107, 2019.

[74] R. P. Chen, B. Liu, H. B. Ni et al., "Vertical flow assays based on core-shell SERS nanotags for multiplex prostate cancer biomarker detection," Analyst, vol. 144, no. 13, pp. 40514059, 2019.

[75] Y. L. Sun, J. F. Fan, L. Y. Cui, W. Ke, F. J. Zheng, and Y. Zhao, "Fluorometric nanoprobes for simultaneous aptamer-based detection of carcinoembryonic antigen and prostate specific antigen," Mikrochimica Acta, vol. 186, no. 3, p. 152, 2019.

[76] J. V. Jokerst and S. S. Gambhir, "Molecular imaging with theranostic nanoparticles," Accounts of Chemical Research, vol. 44, no. 10, pp. 1050-1060, 2011. 
[77] J. Wu, H. J. Lee, L. You et al., "Functionalized NIR-II semiconducting polymer nanoparticles for single-cell to wholeorgan imaging of PSMA-positive prostate cancer," Small, vol. 16, no. 19, article e2001215, 2020.

[78] P. K. Upputuri and M. Pramanik, "Recent advances in photoacoustic contrast agents for in vivo imaging," WIREs Nanomedicine and Nanobiotechnology, vol. 12, no. 4, article e1618, 2020.

[79] G. Cook and V. Goh, "Molecular imaging of bone metastases and their response to therapy," Journal of Nuclear Medicine, vol. 61, no. 6, pp. 799-806, 2020.

[80] M. Salarian, O. Y. Ibhagui, and J. J. Yang, "Molecular imaging of extracellular matrix proteins with targeted probes using magnetic resonance imaging," WIREs Nanomedicine and Nanobiotechnology, vol. 12, no. 4, article e1622, 2020.

[81] V. I. Böhmer, W. Szymanski, K. O. van den Berg et al., "Modular medical imaging agents based on azide-alkyne Huisgen cycloadditions: synthesis and pre-clinical evaluation of $18 \mathrm{~F}-$ Labeled PSMA-tracers for prostate cancer imaging," Chemistry - A European Journal, vol. 26, no. 47, pp. 10871-10881, 2020.

[82] A. Stabile, F. Giganti, V. Kasivisvanathan,, et al., "Factors influencing variability in the performance of multiparametric magnetic resonance imaging in detecting clinically significant prostate cancer: a systematic literature review," European Urology Oncology, vol. 3, no. 2, pp. 145-167, 2020.

[83] P. Kennedy and B. Taouli, "Collagen-targeted MRI contrast agent for liver fibrosis detection," Nature Reviews Gastroenterology \& Hepatology, vol. 17, no. 4, pp. 201-202, 2020.

[84] K. Yamamoto, J. R. Brender, T. Seki et al., "Molecular imaging of the tumor microenvironment reveals the relationship between tumor oxygenation, glucose uptake, and glycolysis in pancreatic ductal adenocarcinoma," Cancer Research, vol. 80, no. 11, pp. 2087-2093, 2020.

[85] L. Wang, H. Yin, R. R. Bi, G. Gao, K. C. Li, and H. L. Liu, "ENO1-targeted superparamagnetic iron oxide nanoparticles for detecting pancreatic cancer by magnetic resonance imaging," Journal of Cellular and Molecular Medicine, vol. 24, no. 10, pp. 5751-5757, 2020.

[86] Y. Huang, L. Li, D. H. Zhang et al., "Gadolinium-doped carbon quantum dots loaded magnetite nanoparticles as a bimodal nanoprobe for both fluorescence and magnetic resonance imaging," Magnetic Resonance Imaging, vol. 68, pp. 113-120, 2020.

[87] D. Psimadas, V. Valotassiou, S. Alexiou, I. Tsougos, and P. Georgoulias, "Radiolabeled mAbs as molecular imaging and/or therapy agents targeting PSMA," Cancer Investigation, vol. 36, no. 2, pp. 118-128, 2018.

[88] S. Lutje, R. Slavik, W. Fendler, K. Herrmann, and M. Eiber, "PSMA ligands in prostate cancer - probe optimization and theranostic applications," Methods, vol. 130, pp. 42-50, 2017.

[89] M. K. Yu, D. Kim, I. H. Lee, J. S. So, Y. Y. Jeong, and S. Jon, "Image-guided prostate cancer therapy using aptamerfunctionalized thermally cross-linked superparamagnetic iron oxide nanoparticles," Small, vol. 7, no. 15, pp. 22412249,2011

[90] K. K. Shung, "Diagnostic ultrasound: past, present, and future," Journal of Medical and Biological Engineering, vol. 31, no. 6, pp. 371-374, 2011.

[91] J. Q. Zhang, Y. H. Chen, C. Deng et al., "The optimized fabrication of a novel nanobubble for tumor imaging," Frontiers in Pharmacology, vol. 10, p. 610, 2019.
[92] G. Wang, L. Song, X. Hou et al., "Surface-modified GVs as nanosized contrast agents for molecular ultrasound imaging of tumor," Biomaterials, vol. 236, article 119803, 2020.

[93] S. A. Boorjian, R. H. Thompson, M. K. Tollefson et al., "Longterm risk of clinical progression after biochemical recurrence following radical prostatectomy: the impact of time from surgery to recurrence," European Urology, vol. 59, no. 6, pp. 893899, 2011.

[94] K. Rahbar, A. Afshar-Oromieh, R. Seifert et al., "Diagnostic performance of 18F-PSMA-1007 PET/CT in patients with biochemical recurrent prostate cancer," European Journal of Nuclear Medicine and Molecular Imaging, vol. 45, no. 12, pp. 2055-2061, 2018.

[95] D. E. Lee, H. Koo, I. C. Sun, J. H. Ryu, K. Kim, and I. C. Kwon, "Multifunctional nanoparticles for multimodal imaging and theragnosis," Chemical Society Reviews, vol. 41, no. 7, pp. 2656-2672, 2012.

[96] Y. T. Wang, C. Chen, Y. Luo,,, et al., "Experimental study of tumor therapy mediated by multimodal imaging based on a biological targeting synergistic agent," International Journal of Nanomedicine, vol. Volume 15, pp. 1871-1888, 2020.

[97] D. W. Hwang, H. Y. Ko, J. H. Lee et al., “A nucleolin-targeted multimodal nanoparticle imaging probe for tracking cancer cells using an aptamer," Journal of Nuclear Medicine, vol. 51, no. 1, pp. 98-105, 2010.

[98] W. J. Kang, J. Lee, Y. S. Lee et al., "Multimodal imaging probe for targeting cancer cells using uMUC-1 aptamer," Colloids and Surfaces B: Biointerfaces, vol. 136, pp. 134-140, 2015.

[99] F. Massari, F. Maines, A. Modena, and M. Brunelli, "Castration resistant prostate cancer (CRPC): state of the art, perspectives and new challenges," Anti-Cancer Agents in Medicinal Chemistry, vol. 13, no. 6, pp. 872-886, 2013.

[100] A. Heidenreich, P. J. Bastian, J. Bellmunt et al., "EAU guidelines on prostate cancer. Part II: treatment of advanced, relapsing, and castration-resistant prostate cancer," European Urology, vol. 65, no. 2, pp. 467-479, 2014.

[101] K. Fizazi, N. Tran, L. Fein et al., "Abiraterone plus prednisone in metastatic, castration-sensitive prostate cancer," The New England Journal of Medicine, vol. 377, no. 4, pp. 352-360, 2017.

[102] C. Parker, S. Nilsson, D. Heinrich et al., "Alpha emitter radium-223 and survival in metastatic prostate cancer," The New England Journal of Medicine, vol. 369, no. 3, pp. 213223, 2013.

[103] M. Shelley, C. Harrison, B. Coles, J. Staffurth, T. J. Wilt, and M. D. Mason, "Chemotherapy for hormone-refractory prostate cancer," Cochrane Database of Systematic Reviews, vol. 4, 2006.

[104] S. Kruspe, F. Mittelberger, K. Szameit, and U. Hahn, “Aptamers as drug delivery vehicles," ChemMedChem, vol. 9, no. 9, pp. 1998-2011, 2014.

[105] Z. Liu, J. H. Duan, and Y. M. Song, "Novel HER2 aptamer selectively delivers cytotoxic drug to HER2-positive breast cancer cells in vitro," Journal of Translational Medicine, vol. 10, no. 1, p. 148, 2012.

[106] H. G. Sun and Y. L. Zu, "Aptamers and their applications in nanomedicine," Small, vol. 11, no. 20, pp. 2352-2364, 2015.

[107] D. Kalyane, N. Raval, R. Maheshwari, V. Tambe, K. Kalia, and R. K. Tekade, "Employment of enhanced permeability and retention effect (EPR): nanoparticle- based precision tools for targeting of therapeutic and diagnostic agent in 
cancer," Materials Science \& Engineering. C, Materials for Biological Applications, vol. 98, pp. 1252-1276, 2019.

[108] S. Catuogno, C. L. Esposito, and V. de Franciscis, “Aptamermediated targeted delivery of therapeutics: an update," Pharmaceuticals, vol. 9, no. 4, p. 69, 2016.

[109] Z. J. Chen, Z. G. Tai, F. F. Gu, C. L. Hu, Q. G. Zhu, and S. Gao, "Aptamer-mediated delivery of docetaxel to prostate cancer through polymeric nanoparticles for enhancement of antitumor efficacy," European Journal of Pharmaceutics and Biopharmaceutics, vol. 107, pp. 130-141, 2016.

[110] X. Wu, Z. G. Tai, Q. G. Zhu, et al., "Study on the prostate cancer-targeting mechanism of aptamer-modified nanoparticles and their potential anticancer effect in vivo," International Journal of Nanomedicine, vol. 9, pp. 5431-5440, 2014.

[111] L. Li, Z. Y. He, X. W. Wei, G. P. Gao, and Y. Q. Wei, "Challenges in CRISPR/CAS9 delivery: potential roles of nonviral vectors," Human Gene Therapy, vol. 26, no. 7, pp. 452-462, 2015.

[112] A. S. Garanina, V. A. Naumenko, A. A. Nikitin et al., "Temperature-controlled magnetic nanoparticles hyperthermia inhibits primary tumor growth and metastases dissemination," Nanomedicine: Nanotechnology, Biology and Medicine, vol. 25, article 102171, 2020.

[113] M.-H. Falk and R.-D. Issels, "Hyperthermia in oncology," International Journal of Hyperthermia, vol. 17, no. 1, pp. 1$18,2001$.

[114] A. Ressel, C. Weiss, and T. Feyerabend, "Tumor oxygenation after radiotherapy, chemotherapy, and/or hyperthermia predicts tumor free survival," International Journal of Radiation Oncology • Biology • Physics, vol. 49, no. 4, pp. 1119-1125, 2001.

[115] B. Hildebrandt, P. Wust, T. Kerner, and R. Felix, "The cellular and molecular basis of hyperthermia," Critical Reviews in Oncology/Hematology, vol. 43, no. 1, pp. 33-56, 2002.

[116] S. Luo, L. F. Wang, W. J. Ding, and H. Wang, "Clinical trials of magnetic induction hyperthermia for treatment of tumours," OA Cancer, vol. 2, no. 1, pp. 1-6, 2014.

[117] M. Johannsen, U. Gneveckow, K. Taymoorian et al., "Morbidity and quality of life during thermotherapy using magnetic nanoparticles in locally recurrent prostate cancer: results of a prospective phase I trial," International Journal of Hyperthermia, vol. 23, no. 3, pp. 315-323, 2007.

[118] M. Johannsen, B. Thiesen, U. Gneveckow et al., "Thermotherapy using magnetic nanoparticles combined with external radiation in an orthotopic rat model of prostate cancer," The Prostate, vol. 66, no. 1, pp. 97-104, 2006.

[119] S. A. McFarland, A. Mandel, R. Dumoulin-White, and G. Gasser, "Metal-based photosensitizers for photodynamic therapy: the future of multimodal oncology?," Current Opinion in Chemical Biology, vol. 56, pp. 23-27, 2020.

[120] X. Mu, S. L. Gan, Y. Wang, H. Li, and G. F. Zhou, "Stimulusresponsive vesicular polymer nano-integrators for drug and gene delivery," International Journal of Nanomedicine, vol. Volume 14, pp. 5415-5434, 2019.

[121] E. Frei, "Combination cancer therapy: presidential address," Cancer Res, vol. 32, no. 12, pp. 2593-2607, 1972.

[122] L. Zhang, A. F. Radovic-Moreno, F. Alexis et al., "Co-delivery of hydrophobic and hydrophilic drugs from nanoparticleaptamer bioconjugates," ChemMedChem, vol. 2, no. 9, pp. 1268-1271, 2007.
[123] N. Wiradharma, Y. W. Tong, and Y. Y. Yang, "Self-assembled oligopeptide nanostructures for co-delivery of drug and gene with synergistic therapeutic effect," Biomaterials, vol. 30, no. 17, pp. 3100-3109, 2009.

[124] K. Nagamatsu, F. Tsuchiya, K. Oguma, H. Maruyama, R. Kano, and A. Hasegawa, "The effect of small interfering RNA (siRNA) against the Bcl-2 gene on apoptosis and chemosensitivity in a canine mammary gland tumor cell line," Research in Veterinary Science, vol. 84, no. 1, pp. 49-55, 2008.

[125] X. L. Liu, Y. F. Zhang, Y. Y. Wang, et al., "Comprehensive understanding of magnetic hyperthermia for improving antitumor therapeutic efficacy," Theranostics, vol. 10, no. 8, pp. 3793-3815, 2020.

[126] F. F. Zhao, J. Zhou, X. J. Su et al., “A smart responsive dual aptamers-targeted bubble-generating nanosystem for cancer triplex therapy and ultrasound imaging," Small, vol. 13, no. 20, pp. 1-13, 2017.

[127] S. Soundararajan, W. W. Chen, E. K. Spicer, N. CourtenayLuck, and D. J. Fernandes, "The nucleolin targeting aptamer AS1411 destabilizes Bcl-2 messenger RNA in human breast cancer cells," Cancer Research, vol. 68, no. 7, pp. 23582365, 2008.

[128] Y. Lin, J. Chen, and B. Shen, "Interactions between genetics, lifestyle, and environmental factors for healthcare," Advances in Experimental Medicine and Biology, vol. 1005, pp. 167191, 2017.

[129] D. Karan, J. B. Thrasher, and D. Lubaroff, "Prostate cancer: genes, environment, immunity and the use of immunotherapy," Prostate Cancer and Prostatic Diseases, vol. 11, no. 3, pp. 230-236, 2008.

[130] P. Larsson, A. S. Syed Khaja, J. Semenas et al., "The functional interlink between AR and MMP9/VEGF signaling axis is mediated through PIP5K1 $\alpha /$ pAKT in prostate cancer," International Journal of Cancer, vol. 146, no. 6, pp. 1686-1699, 2020.

[131] C. J. Paller, D. Piana, J. R. Eshleman, et al., "A pilot study of prostate-specific membrane antigen (PSMA) dynamics in men undergoing treatment for advanced prostate cancer," The Prostate, vol. 79, no. 14, pp. 1597-1603, 2019.

[132] S. Y. Ku, M. E. Gleave, and H. Beltran, "Towards precision oncology in advanced prostate cancer," Nature Reviews. Urology, vol. 16, no. 11, pp. 645-654, 2019.

[133] Y. X. Lin, X. J. Zhao, Z. J. Miao et al., "Data-driven translational prostate cancer research: from biomarker discovery to clinical decision," Journal of Translational Medicine, vol. 18, no. 1, p. 119, 2020. 